\title{
Linear Algebra
}

Linear Algebra is a huge subject nowadays and also touches a gamut of applications, ranging from representation theory in pure group theory to the most advanced computational science. Here in Appendices A.1 and A.2 I very briefly bring to mind the contexts of Matrix Analysis and Operator Theory as I see them, thereby helping to explain the flavors of this book.

\section{A.1 Matrix Analysis}

A typical first semester undergraduate Linear Algebra course should start with matrix-vector multiplication and teach the Gauss reduction procedure to solve linear systems. One should then introduce the axioms of vector spaces and of algebras and show that the $n \times n$ matrices are both. Then the Fundamental Theorem of Linear Algebra, that $R(A)=N\left(A^{*}\right)^{\perp}$, should be established. Next, eigenvalue-eigenvector problems should be posed and solved for both symmetric and nonsymmetric matrices $A$. Finally, the singular value decomposition, and as many of its ramifications as time permits, should be given ample study.

The next (graduate) course should immediately emphasize how to change bases, while at the same time stressing the need to pick a good one to calculate anything useful. Schur's upper-triangularization theorem and the Jordan canonical form should be proved. I might next go to computational linear solvers, but others teaching such a course could go to other topics, for example search engines. They are too many to be listed here.

What are good textbooks? There are so many good ones out there that when publishers approached me to write my own, I decided not to. What have I found myself choosing as textbook? I have chosen the undergraduate text by D. Lay, Linear Algebra and its Applications, and the graduate text by 
R. Horn and C. Johnson, Matrix Analysis. Both of these excellent books have proven themselves to wide audiences.

\section{A.2 Operator Theory}

I was raised on S. Goldberg's little classic, Unbounded Linear Operators, although before that I had been exposed to the classic Functional Analysis by F. Riesz and B. S. Nagy. Of course I like Kato's Perturbation Theory for Linear Operators. When I taught a course in functional analysis many years ago I found myself choosing Yosida's Functional Analysis. It would appear that I prefer the older classics to any of the newer texts I have chanced upon. However, I am sure there are some good new ones. If I were to teach such course again, I would probably not be able to resist P. Lax's Functional Analysis, just because of its interesting content.

How do I see operator theory? Because of my background in partial differential equations and its applications, I see unbounded operators in a Hilbert space as the place to start. Then their resolvent operators $(\lambda I-A)^{-1}$ take you to bounded operators. Then if you actually need to compute things useful in real applications, you will specialize further to finite-dimensional matrices. Quickly you will find yourself immersed in linear solvers for very large but finite matrix problems. You could spend the rest of your life working within the enterprise of linear solvers. I am astonished at some of my colleagues in algebra who do not even know of the huge field of computational linear algebra. At least I have worked with Galois fields for error correction, and with Lie algebras for differential equations. Perhaps Linear Algebra is now the most important topic in Algebra. 


\section{Hints and Answers to Exercises}

\section{Chapter 1.}

1. Schwarz's inequality is perhaps the most important inequality in mathematics, hence, in all the world. If you just care about real Hilbert spaces, I give the following very short proof in my book Partial Differential equations [Gustafson (1999f)]. Let $Q(t)=\langle u+t v, u+t v\rangle=\|u\|^{2}+$ $t(2\langle u, v\rangle)+t^{2}\|v\|^{2}$. As $Q(t)$ is never negative, Schwarz's inequality is just the nonpositivity of $Q(t)^{\prime}$ s discriminant. You will find other inequalities we assume in this book, in that PDE book, and will notice from Chapter 1 that I prefer the notation $\langle x, y\rangle$ for inner product.

2. There are several ways to approach this, notably, by functional minimization, or just using ordinary calculus. Let us do the latter here. Consider $R(x)$ denote the Rayleigh quotient for the matrix $A=\left[a_{i j}\right]$. Let $x_{1}=\left[x_{i}^{1}\right]$ be any lowest eigenvector. Then $R(x)$ is a continuous, even differentiable, function of all of the coordinates $x_{i}$. $R(x)$ achieves its minimum at $x_{1}$. Hence, for $i=1,2, \ldots, n$, we have necessarily the system

$$
\begin{aligned}
0 & =\frac{\partial R(x)}{\partial x_{i}}=\frac{\partial}{\partial x_{i}}\left[\frac{\sum_{i, j=1}^{n} a_{k j} a_{k} x_{j}}{\sum_{i=1}^{n} x_{i}^{2}}\right]_{x_{i}=x_{i}^{1}}=2\left[\frac{\sum_{i=1}^{n} a_{i j} x_{j}^{1}-x_{i}^{1} R\left(x_{1}\right)}{\sum_{k=1}^{n}\left(x_{k}^{1}\right)^{2}}\right] \\
& =A x_{1}-\lambda x_{1} .
\end{aligned}
$$

You may do the higher antieigenvalues the same way on the reduced matrix on the reduced spaces. 
3. Everyone is familiar with the spectral representation

$$
A=\sum_{i=1}^{n} \lambda_{i} x_{i} x_{i}^{*}=\sum_{i=1}^{n} \lambda_{i} P_{i}, \quad P_{i}=P_{i}^{2}=P_{i}^{*},
$$

for selfadjoint matrices. But many do not keep cognizance of the more general theorem expressing $A$ in terms of rank-one outer products

$$
A=\sum_{k=1}^{k} x_{i} y_{i}^{*}
$$

for arbitrary matrices of rank $k$. I like to get my version of this just by a judicious Gauss elimination procedure: Let $E A=E_{k} E_{k-1} \cdots E_{1} A=$ $Y$ be a reduction of $A$ to an upper echelon form $Y$, so that $A=X Y$ where $X=E_{1}^{-1} \cdots E_{k-1}^{-1} E_{k}^{-1}$. Then write the matrix product $X Y$ as outerproducts. Thus $A=X Y=X_{1} Y_{1}^{*}+\cdots+X_{k} Y_{k}^{*}$ where the $X_{i}$ are the columns of $X$ and the $Y_{i}$ the rows of $Y$. Then I like to go further and normalize to weighted projections

$$
A=\sum_{i=1}^{k}\left(y_{i}^{*} x_{i}\right) \frac{x_{i} y_{i}^{*}}{y_{i}^{*} x_{i}}=\sum_{i=1}^{k}\left\langle x_{i}, y_{i}\right\rangle P_{x}^{y^{\perp}} .
$$

Here each $P_{i}$ is the oblique projector onto the span of $x$, projecting from the direction $y^{\perp}$. That is the reason for my notation $P_{x}^{y^{\perp}}$.

Here is an example. Let

$$
x=\left[\begin{array}{l}
1 \\
0
\end{array}\right], \quad y=\left[\begin{array}{l}
1 \\
1
\end{array}\right], \quad w=\left[\begin{array}{c}
1 \\
1 / 2
\end{array}\right] .
$$

Then for any $x, y, w$, we have the projector implemented in either of two ways:

$$
P_{x}^{y^{\perp}} w=\frac{x y^{*}}{y^{*} x}(w)=\frac{\left(y^{*} w\right)}{\left(y^{*} x\right)} x
$$

For the specific vectors $x, y$, and $w$ above, we have

$$
P_{x}^{y^{\perp}}(w)=\left[\begin{array}{ll}
1 & 1 \\
0 & 0
\end{array}\right]\left[\begin{array}{c}
1 \\
1 / 2
\end{array}\right]=\left[\begin{array}{c}
3 / 2 \\
0
\end{array}\right]
$$

and

$$
P_{x}^{y^{\perp}}(w)=\left(\left[\begin{array}{ll}
1 & 1
\end{array}\right]\left[\begin{array}{c}
1 \\
1 / 2
\end{array}\right]\right)\left[\begin{array}{l}
1 \\
0
\end{array}\right]=\left[\begin{array}{c}
3 / 2 \\
0
\end{array}\right] .
$$


It is nice to draw the picture of this simple example.

One need not use my Gauss elimination recipe above, and indeed the possibilities for such oblique projections are rich. How you choose to do will depend upon your needs. For example, one may just spectrally project onto the columns of a matrix $A$, e.g.,

$$
A=\left[\begin{array}{ll}
1 & 2 \\
3 & 1
\end{array}\right]=\left[\begin{array}{ll}
1 & 0 \\
3 & 0
\end{array}\right]+\left[\begin{array}{ll}
0 & 2 \\
0 & 1
\end{array}\right]=\left[\begin{array}{l}
1 \\
3
\end{array}\right]\left[\begin{array}{ll}
1 & 0
\end{array}\right]+\left[\begin{array}{l}
2 \\
1
\end{array}\right]\left[\begin{array}{ll}
0 & 1
\end{array}\right] .
$$

Orthogonal projectors $P$ have long ruled for many good reasons. We like to think in terms of the natural basis $\left\{e_{i}\right\}$. Generally they provide better round-off accuracy because they have $\|P\|=1$. But as we move more and more into general matrices, oblique projectors will more often naturally occur in their own right.

4. My favorite book on unbounded operator theory is [Kato (1976)].

5. This is even true in infinite dimensions. See [Halmos (1967)].

6. This is an infinite-time problem, so just follow your interests.

\section{Chapter 2.}

1. One may employ Green's identity

$$
0 \leqq \int_{\Omega}(\operatorname{grad} u)^{2}=\oint_{\partial \Omega} u \frac{\partial u}{\partial n}-\int_{\Omega} u \Delta u,
$$

that holds for smooth functions $u$. Then you must go to the full domain $W^{2,2}(\Omega)$ of the operator $A$.

On bounded domains $\Omega$ one can show that the operator $-\Delta$ is strongly accretive. The Rayleigh quotient for functions $u$ that vanish on the boundary $\partial \Omega$ is nonnegative, i.e.,

$$
\frac{\langle-\Delta u, u\rangle}{\langle u, u\rangle}=\frac{\int_{\Omega}(\operatorname{grad} u)^{2}}{\int_{\Omega} u^{2}} \geqq 0 .
$$

Suppose it is zero for some $u$ in the domain of the operator. Then the gradient $\operatorname{grad} u$ is zero at each point in $\Omega$, hence $u$ is constant over $\Omega$, and hence by continuity to the boundary, $u$ is zero on the closed domain $\bar{\Omega}$, a contradiction. Stated another way, the operator is selfadjoint with positive lowest eigenvalue $\lambda_{1}$. We have glossed over the need to take this argument from a smooth dense subspace of functions $u$ to the whole domain of the operator. 
2. See any ordinary differential equations textbook.

3. For an overly brief account, see the Epilogue in my PDE book [Gustafson (1999f)].

4. See my book [Gustafson (1999f)]. For a much more comprehensive treatment see [Kato (1976)].

5. For $A$ an unbounded operator, the statement that $A$ commutes with bounded everywhere defined $B$ means the operator product inclusion

$$
B A \subset A B \text {. }
$$

The domain of the left-hand side is $D(A)$. Normally the domain of $A B$ depends on the meshing of the range $R(B)$ with the domain $D(A)$. So a commuting assumption is even stronger than its face value would indicate. In particular, it means that $D(A B)$ is at least as large as $D(A)$.

$A$ possesses positive square root $A^{1 / 2}$ such that

$$
B A^{1 / 2} \subset A^{1 / 2} B \text {. }
$$

Thus $\langle B A x, x\rangle=\left\langle B A^{1 / 2} x, A^{1 / 2} x\right\rangle$ on $D(B A)=D(A) \subset D\left(A^{1 / 2}\right)$. Hence $\operatorname{Re}\langle B A x, x\rangle \geqq 0$ due to the accretivity of $B$.

As to the second assertion, first we establish that $B A$ is symmetric. For bounded operators $B$, one always has the adjoint inclusion relation $(B A)^{*} \supset A^{*} B^{*}$. For the case at hand, from $B A \subset A B$ we have

$$
(B A)^{*}=A B^{*} \supset(A B)^{*} \supset B^{*} A .
$$

Thus in particular, $A$ also commutes with $B^{*}$. If we now impose the stronger hypothesis that $B$ is selfadjoint and strongly positive, then the inclusion above becomes

$$
(B A)^{*}=A B \supset(A B)^{*} \supset B A
$$

and therefore $B A$ is a symmetric operator.

By the first part of the problem, $B A$ is already accretive. If $B A$ is moreover selfadjoint, then its accretivity turns into positivity (nonnegativity to be more precise). That turns into strong positivity from the strong positivity of $B$ and that of $A$, hence of $A^{1 / 2}$ : for $x$ in $D(A)$ we have

$$
\begin{aligned}
\langle B A x, x\rangle & =\left\langle B A^{1 / 2} x, A^{1 / 2} x\right\rangle \geqq m_{B}\left\langle A^{1 / 2} x, A^{1 / 2} x\right\rangle \\
& =m_{B}\langle A x, x\rangle \geqq m_{B} m_{A}\langle x, x\rangle .
\end{aligned}
$$

I have used the fact that when we say a selfadjoint operator is positive, that implicitly implies that $A$ is strongly positive. That is the case when 
$A$ is bounded, but probably for unbounded $A$ one should more carefully just make the assumption of strong positivity.

In any case, it remains to show that $B A$ is selfadjoint. For this we may use the Rellich-Kato additive perturbation theorem. For small positive $\epsilon$, write as I did in the multiplicative perturbation proofs

$$
\epsilon B A=(\epsilon B-I) A+A .
$$

Since $\|\epsilon B-I\|=b<1, A$ is selfadjoint and the perturbation $(\epsilon B-I) A$ is symmetric, by the Rellich-Kato Theorem the right-hand side is selfadjoint. No doubt there are other proofs but I wanted an exercise on adjointing and commuting of unbounded operators and a use of the Rellich-Kato Theorem.

6. See the paper [Gustafson (2007a)]. As I recall at this writing, I have not gotten around to the next step, namely, of developing the operator trigonometry which might result by utilizing this graph norm-to-norm unbounded closed operators.

\section{Chapter 3.}

1. There are many proofs of this theorem in the literature. I like my own, [Gustafson (1970a)], because it uses the same mindset as that which I used earlier in proving my Min-Max Theorem [Gustafson (1968d)].

2. The corrected expression on p. 163 of [Gustafson (1968c)] is

$$
\|\epsilon B+I\| \leqq\left\|I-\epsilon^{2} B^{2}\right\| \cdot\left\|(\epsilon B-I)^{-1}\right\| \leqq\left(1+\epsilon^{2}\|B\|^{2}\right) \cdot(1-\epsilon \theta(B))^{-1} .
$$

3. Let $\mathbb{R}^{2}$ have a "skew" unit ball. For a concrete example, draw the unit ball as follows. Starting at the point $\left(x_{1}, x_{2}\right)=(-1,-1)$, come horizontally to the right to $\left(x_{1}, x_{2}\right)=(1 / 2,-1)$, then go up to the right at $60^{\circ}$ to the point $\left(x_{1}, x_{2}\right)=(1,0)$, go straight up to $\left(x_{1}, x_{2}\right)=(1,1)$, turn horizontally left all the way back to $\left(x_{1}, x_{2}\right)=(-1 / 2,1)$, descend at $60^{\circ}$ to the $x_{1}$ axis at $\left(x_{1}, x_{2}\right)=(-1,0)$, then drop vertically down to the first point $\left(x_{1}, x_{2}\right)=(-1,-1)$.

Now let $B$ be the $2 \times 2$ matrix $B=\left[\begin{array}{cc}1 / 2 & 0 \\ 0 & 1\end{array}\right]$. Then letting $\epsilon$ trace through the values $\epsilon=1,5 / 4,3 / 2$, one may verify that the $\|\epsilon B-I\|$ curve is exactly the line $1-\epsilon / 2$ for $0 \leqq \epsilon \leqq 1$, then the flat of value $1 / 2$ for $1 \leqq \epsilon \leqq 3 / 2$, then the increasing line $-1+\epsilon$ for $\epsilon \geqq 3 / 2$. 
4. From $F(\lambda)=\left(2+\lambda^{2}\right) /\left(\lambda^{4}+5 \lambda^{2}+4\right)^{1 / 2}$ we obtain

$$
F^{\prime}(\lambda)=\frac{\lambda^{3}-2 \lambda}{\left(\lambda^{4}+5 \lambda^{2}+4\right)^{3 / 2}}
$$

and

$$
F^{\prime \prime}(\lambda)=\frac{\left(\lambda^{4}+5 \lambda^{2}+4\right)\left(3 \lambda^{2}-2\right)-3\left(\lambda^{3}-2 \lambda\right)\left(4 \lambda^{3}+10 \lambda\right) / 2}{\left(\lambda^{4}+5 \lambda^{2}+4\right)^{5 / 2}} .
$$

$F^{\prime}(\lambda)=0$ at $\lambda=0$ or $\lambda= \pm \sqrt{2}$. The former comes from the largest eigenvector which creates the $F(\lambda)=1$ maximum at $\lambda=0$. The latter come from the two antieigenvectors and create the minima as shown in Fig. 3.2. To obtain the inflection points we set the numerator of $F^{\prime \prime}(\lambda)=0$ from which we obtain the sixth-order polynomial equation

$$
3 \lambda^{6}-10 \lambda^{4}-32 \lambda^{2}+8=0 .
$$

I could not quickly factor this so I went to the computer to find the approximate positive roots $\lambda^{2}=0.23408$ and 5.26358, whose square roots provide the inflection points for Fig. 3.2.

As $\lambda \rightarrow \infty$ we see that

$$
F(\lambda)=\frac{1+2 / \lambda^{2}}{1+5 / \lambda^{2}+4 / \lambda^{4}} \rightarrow 1
$$

and

$$
F^{\prime}(\lambda)=\frac{1-2 / \lambda^{2}}{\left(\lambda^{2}+5+4 / \lambda^{2}\right)^{3 / 2}} \rightarrow 0 .
$$

5. You should start from scratch by putting your $x_{ \pm}^{j k}$ directly into the Euler equation. However, starting from our expressions in proving the converse, you have immediately that

$$
\begin{aligned}
& \left|c_{1}\right|^{2} \lambda_{1}^{2}+\left|c_{2}\right|^{2} \lambda_{2}^{2}=\lambda_{2} \lambda_{1}, \\
& \left|c_{1}\right|^{2} \lambda_{1}+\left|c_{2}\right|^{2} \lambda_{2}=\frac{2 \lambda_{2} \lambda_{1}}{\lambda_{1}+\lambda_{2}},
\end{aligned}
$$

and hence the coefficient of $x_{1}$ becomes simply

$$
\frac{\lambda_{1}}{\lambda_{2}}-\frac{2\left(\lambda_{1}+\lambda_{2}\right)}{2 \lambda_{2}}+1=0 .
$$

Similarly for the other coefficient.

6. I must confess that I have not worked out the particulars. And there may be interesting unforseen consequences in considering threecomponent and $k$-component generalizations of my antieigenvectors. However, keep in mind that physical intuition says that if you try to 
turn a three-component vector $x$ with $A$, the "middle" component will get in the way and you will therefore turn more by dropping the middle component from the trial vector. So one needs to look for trigonometrical features other than just maximum turning dynamics.

\section{Chapter 4.}

1. D. M. Young is the older and very famous computational linear algebraist from Texas and Ph.D. student of G. Birkhoff at Harvard. His book which really put iterative methods on the map and which contributed SOR to our mathematics is D. M. Young, Iterative Solutions of Large Linear Systems, Academic Press, 1971.

D. P. Young was my Ph.D. student (1979) at Colorado. He was so good that Boeing snapped him up immediately, and to my knowledge David is still there and has been a key design engineer for CFD projects such as the Space Shuttle, Stealth, and Boeing 777.

I first met the senior D. M. Young at the Nijmegen conference in 1996. He humorously castigated me for the resulting confusion caused by my bringing another D. Young into the computational linear algebra literature!

2.

$$
\sin ^{2} \phi\left(A^{1 / 2}\right)=\left[\frac{\lambda_{n}^{1 / 2}-\lambda_{1}^{1 / 2}}{\lambda_{n}^{1 / 2}+\lambda_{1}^{1 / 2}}\right]^{2}=\frac{\lambda_{n}+\lambda_{1}-2 \lambda_{n}^{1 / 2} \lambda_{1}^{1 / 2}}{\lambda_{n}+\lambda_{1}+2 \lambda_{n}^{1 / 2} \lambda_{1}^{1 / 2}} .
$$

Thus

$$
\sin \phi\left(A^{1 / 2}\right)=\left[\frac{1-\cos \phi(A)}{1+\cos \phi(A)}\right]^{1 / 2}=\frac{\sin \phi(A)}{1+\cos \phi(A)} .
$$

3. See [Gustafson (1998g)] for details. Block ordering $A$ produced a better angular route to the solution.

4. The eigenvalues of $A_{h}$ are

$$
\lambda_{i j}=4 h^{-2}\left[\sin ^{2}\left(\frac{i \pi h}{2}\right)+\sin ^{2}\left(\frac{j \pi h}{2}\right)\right], \quad 1 \leqq i, \quad j \leqq N-1 .
$$

The corresponding $i, j$-th eigenvectors $e^{i j}$ are the $(N-1)^{2}$ dimension vectors with components

$$
\left(e^{i j}\right)_{k \ell}=2 h \sin (i \pi h k) \sin (j \pi h \ell),
$$

with $1 \leqq k, \ell \leqq N-1$ taken in natural order. 
Thus the smallest and largest eigenvalues are

$$
\begin{gathered}
\lambda_{1}=\lambda_{11}=8 h^{-2} \sin ^{2}\left(\frac{\pi h}{2}\right), \\
\lambda_{n}=\lambda_{N-1, N-1}=8 h^{-2} \sin ^{2}\left(\frac{(N-1) \pi h}{2}\right) \\
=8 h^{-2} \cos ^{2}\left(\frac{\pi h}{2}\right) .
\end{gathered}
$$

For the coefficients of the antieigenvectors we need

$$
\begin{aligned}
& \left(\frac{\lambda_{n}}{\lambda_{1}+\lambda_{n}}\right)^{1 / 2}=\frac{\cos (\pi h / 2)}{\left(\sin ^{2}(\pi h / 2)+\cos ^{2}(\pi h / 2)\right)^{1 / 2}}=\cos \left(\frac{\pi h}{2}\right), \\
& \left(\frac{\lambda_{1}}{\lambda_{1}+\lambda_{n}}\right)^{1 / 2}=\frac{\sin (\pi h / 2)}{\left(\sin ^{2}(\pi h / 2)+\cos ^{2}(\pi h / 2)\right)^{1 / 2}}=\sin \left(\frac{\pi h}{2}\right) .
\end{aligned}
$$

Thus

$$
x_{ \pm}= \pm \cos \left(\frac{\pi h}{2}\right) e_{11}+\sin \left(\frac{\pi h}{2}\right) e_{N-1, N-1} .
$$

For the $4 \times 4$ coarse mesh example with $h=1 / 3$ one has

$$
\begin{aligned}
& \lambda_{11}= \frac{8}{9} \sin ^{2}\left(\frac{\pi}{6}\right)=\frac{2}{9}, \\
& \lambda_{22}=\frac{8}{9} \cos ^{2}\left(\frac{\pi}{6}\right)=\frac{6}{9} \\
& e_{11}=\frac{2}{3}\left[\begin{array}{c}
\sin \left(\frac{\pi}{3}\right) \sin \left(\frac{\pi}{3}\right) \\
\sin \left(\frac{2 \pi}{3}\right) \sin \left(\frac{\pi}{3}\right) \\
\sin \left(\frac{\pi}{3}\right) \sin \left(\frac{2 \pi}{3}\right) \\
\sin \left(\frac{2 \pi}{3}\right) \sin \left(\frac{2 \pi}{3}\right)
\end{array}\right]=\frac{1}{2}\left[\begin{array}{c}
1 \\
1 \\
1 \\
1
\end{array}\right], \\
& e_{22}=\frac{2}{3}\left[\begin{array}{l}
\sin \left(\frac{2 \pi}{3}\right) \sin \left(\frac{2 \pi}{3}\right) \\
\sin \left(\frac{4 \pi}{3}\right) \sin \left(\frac{2 \pi}{3}\right) \\
\sin \left(\frac{2 \pi}{3}\right) \sin \left(\frac{4 \pi}{3}\right) \\
\sin \left(\frac{4 \pi}{3}\right) \sin \left(\frac{4 \pi}{3}\right)
\end{array}\right]=\frac{1}{2}\left[\begin{array}{c}
1 \\
-1 \\
-1 \\
1
\end{array}\right] .
\end{aligned}
$$


From these we obtain

$$
x_{ \pm}= \pm\left(\frac{6}{2+6}\right)^{1 / 2} e_{11}+\left(\frac{2}{2+6}\right)^{1 / 2} e_{22}= \pm\left(\frac{\sqrt{3}}{2}\right) e_{11}+\frac{1}{2} e_{22}
$$

which more specifically are

$$
x_{+}=\frac{1}{4}\left[\begin{array}{l}
\sqrt{3}+1 \\
\sqrt{3}-1 \\
\sqrt{3}-1 \\
\sqrt{3}+1
\end{array}\right] \cong\left[\begin{array}{l}
0.68301 \\
0.18301 \\
0.18301 \\
0.68301
\end{array}\right]
$$

and

$$
x_{-}=\frac{1}{4}\left[\begin{array}{l}
-\sqrt{3}+1 \\
-\sqrt{3}-1 \\
-\sqrt{3}-1 \\
-\sqrt{3}+1
\end{array}\right] \cong\left[\begin{array}{l}
-0.18301 \\
-0.68301 \\
-0.68301 \\
-0.18301
\end{array}\right] .
$$

5. Starting from the result of Chatelin and Gratton (2000), we have

$$
\begin{aligned}
C^{2}(H) & =\frac{2\left(1+\kappa^{2}(A)\right)}{(1+\kappa(A))^{2}}=\frac{2\left(1+\sigma_{1}^{2}(A) / \sigma_{n}^{2}(A)\right)}{\left(1+\sigma_{1}(A) / \sigma_{n}(A)\right)^{2}} \\
& =\frac{2\left(\lambda_{1}^{2}(H)+\lambda_{n}^{2}(H)\right)}{\left(\lambda_{1}(H)+\lambda_{n}(H)\right)^{2}}=1+\left(\frac{\lambda_{n}(H)-\lambda_{1}(H)}{\lambda_{n}(H)+\lambda_{1}(H)}\right)^{2} .
\end{aligned}
$$

6. Hint: See [Gustafson (2004a)]. There I characterized the positive cone $\{C\}$ by a Jordan canonical form analysis in which I wanted $A B$ in its upper Jordan form $\widetilde{A B}$. To get there for the $2 \times 2$ example of the problem at hand, note that

$$
B A=\left[\begin{array}{cc}
1 & 2 / 7 \\
1 / 2 & 1
\end{array}\right], \quad A B=\left[\begin{array}{cc}
1 & 1 / 2 \\
2 / 7 & 1
\end{array}\right]
$$

and $\sigma(A B)=\sigma(B A)$ are the eigenvalues

$$
\lambda_{1}=1-\frac{1}{7}, \quad \lambda_{2}=1+\frac{1}{7} .
$$

Seeking all similarity matrices $M$ such that $(A B) M=M(\widetilde{A B})$ means we want

$$
\left[\begin{array}{cc}
1 & 1 / 2 \\
2 / 7 & 1
\end{array}\right]\left[\begin{array}{ll}
m_{11} & m_{12} \\
m_{21} & m_{22}
\end{array}\right]=\left[\begin{array}{cc}
m_{11} & m_{12} \\
m_{21} & v_{22}
\end{array}\right]\left[\begin{array}{cc}
1-\sqrt{7} / 7 & 0 \\
0 & 1+\sqrt{7} / 7
\end{array}\right] .
$$


One eventually arrives at the expression

$$
C=\left[\begin{array}{cc}
m_{11}^{2} \lambda_{1}+m_{22}^{2} \lambda_{2}(7 / 4) & m_{11}^{2} \lambda_{1}(-2 / \sqrt{7})+m_{22}^{2} \lambda_{2}(\sqrt{7} / 2) \\
m_{11}^{2} \lambda_{1}(-2 / \sqrt{7})+m_{22}^{2} \lambda_{2}(\sqrt{7} / 2) & m_{11}^{2} \lambda_{1}(4 / 7)+m_{22} \lambda_{2}
\end{array}\right] .
$$

See [Gustafson (2004a)] for details.

\section{Chapter 5.}

1. Recall that our Time-operator for any wavelet is

$$
T f=\sum_{m \in \mathbb{Z}} \sum_{n \in \mathbb{Z}} m\left\langle\psi_{m n}, f\right\rangle \psi_{m n}(x) .
$$

The Haar wavelet $\psi_{1,1}$ oscillates once with amplitude $2^{-3 / 2}$ on its support $[2,4]$ and the wavelet $\psi_{3,0}$ oscillates once with amplitude $2^{-3 / 2}$ on its support $[0,8)$. Our new example of a wavelet twisting vector will be my antieigenvector (I just do the $x_{+}$positive version here), which becomes

$$
\begin{aligned}
\psi_{+} & =\left(\frac{m_{\text {older }}}{m_{\text {older }}+m_{\text {younger }}}\right)^{1 / 2} \psi_{\text {younger }}+\left(\frac{m_{\text {younger }}}{m_{\text {older }}+m_{\text {younger }}}\right)^{1 / 2} \psi_{\text {older }} \\
& =\left(\frac{3}{4}\right)^{1 / 2} \psi_{1,1}+\left(\frac{1}{4}\right)^{1 / 2} \psi_{3,0}=\frac{1}{2}\left[\sqrt{3} \psi_{1,1}+\psi_{3,0}\right] .
\end{aligned}
$$

By hand calculator I sketched out this function $\psi_{+}$on the interval $[0,8)$ by finding it to be approximately: $\psi=0.176$ on [0, 2), 0.788 on $[2,3)$, -0.436 on $[3,4)$, and -0.0176 on $[4,8)$. It produces an age turning angle of $30^{\circ}$, because

$$
\sin \phi\left(\psi_{1,1}, \psi_{3,0}\right)=\frac{m_{\text {older }}-m_{\text {younger }}}{m_{\text {older }}+m_{\text {younger }}}=\frac{3-1}{3+1}=\frac{1}{2} .
$$

You should do the same for $\psi_{-}$.

2. See the bibliographies in our papers.

3. $S$ was defined by $S x=\sum_{n}\left\langle x, x_{n}\right\rangle x_{n}$ so first we must assure that $S$ is an operator, i.e., that the series defining it converges. But the partial sums can be seen to be a Cauchy sequence:

$$
\left\|\sum^{N}\left\langle x, x_{n}\right\rangle x_{n}-\sum^{M}\left\langle x, x_{n}\right\rangle x_{n}\right\|^{2}=\left\|\sum_{N}^{M}\left\langle x, x_{n}\right\rangle x_{n}\right\|^{2} \leqq B \sum_{N}^{M}\left|\left\langle x, x_{n}\right\rangle\right|^{2}
$$


which by the upper frame bound must go to zero. Then we may look at the quadratic form view of $S$, namely, $\langle S x, x\rangle$, and see that

$$
\begin{aligned}
\langle S x, x\rangle & =\left\langle\sum_{n}\left\langle x, x_{n}\right\rangle x_{n}, x\right\rangle=\sum_{n}\left\langle x, x_{n}\right\rangle\left\langle x_{n}, x\right\rangle \\
& =\sum_{n}\left|\left\langle x, x_{n}\right\rangle\right|^{2} .
\end{aligned}
$$

Thus we may simply rewrite the original frame as

$$
A\|x\|^{2} \leqq\langle S x, x\rangle \leqq B\|x\|^{2} .
$$

From this by standard operator theory we know that

$$
B^{-1}\|x\|^{2} \leqq\left\langle S^{-1} x, x\right\rangle \leqq A^{-1}\|x\|^{2} .
$$

But $\left\langle S^{-1} x, x\right\rangle=\sum_{n}\left|\left\langle x, x_{n}\right\rangle\right|^{2}$ by the above reasoning so $\left\{S^{-1} x_{n}\right\}$ is a frame.

4. It is better to think of $S$ in its sesquilinear form bounds than in its operator bounds, although since $S$ is SPD, they are equivalent. Recall our situation, stated here somewhat redundantly:

$$
0<A \leqq\left\|S^{-1}\right\|^{-1}=m_{S} \leqq S \leqq M_{S}=\|S\| \leqq B .
$$

From this we have as operator forms, for any $\epsilon>0$,

$$
-I \leqq \epsilon A-I \leqq \epsilon m_{S}-I \leqq \epsilon S-I \leqq \epsilon M_{S}-I \leqq \epsilon B-I .
$$

To keep everything here positive so that we may automatically convert to operator norms, we impose lower bound $\epsilon>1 / A$. To keep everything bounded above by 1 , we impose upper bound $\epsilon<2 / B$. For that interval $A^{-1}<\epsilon<2 B^{-1}$ we are assured the norm curve satisfies

$$
0<\|\epsilon S-I\|<1 .
$$

However, we already know generally that $\|\epsilon S-I\|$ is less than one for all $0<\epsilon<2 B^{-1}$, so the $A^{-1}$ lower bound is not needed to keep $\|\epsilon S-I\|$ less than one. On the other hand we know that $\epsilon_{m}=2 /\left(m_{S}+M_{S}\right)$ exactly. So from the same form inequalities above we have the bound $A^{-1}<\epsilon_{m}<B^{-1}$ for the minimizing $\epsilon_{m}$. Notice that if $A$ and $B$ were exact, then we would know that $\epsilon_{m}=2 /(A+B)$, so the bound $A^{-1}<$ $\epsilon_{m}<B^{-1}$ then becomes $A>(A+B) / 2>B$, which is just the arithmetic mean inequality.

5. I leave it to the reader to play trigonometrically (in the conventional trigonometric sense) with some of these new Basis Trigonometry considerations and examples.

6. Dropping the normalization factor four for now, we have

$$
\psi_{+}=3 \sin x-\sin 3 x,
$$




$$
\begin{aligned}
A \psi_{+} & =-\psi_{+}^{\prime \prime}=3 \sin x-9 \sin 3 x, \\
\left\|A \psi_{+}\right\|^{2} & =9\|\sin x\|^{2}+81\|\sin 3 x\|^{2}=45 \pi, \\
\left\|A \psi_{+}\right\| & =3 \pi^{1 / 2} \sqrt{5}, \\
\left\|\psi_{+}\right\|^{2} & =9\|\sin x\|^{2}+\|\sin 3 x\|^{2}=5 \pi, \\
\left\|\psi_{+}\right\| & =\pi^{1 / 2} \sqrt{5} .
\end{aligned}
$$

Thus the antieigenvalue quotient $\mu\left(\psi_{+}\right)$is

$$
\mu\left(\psi_{+}\right)=\frac{\langle A x, x\rangle}{\|A x\|\|x\|}=\frac{9\|\sin x\|^{2}+9\|\sin 3 x\|^{2}}{15 \pi}=\frac{9 \pi}{15 \pi}=0.6
$$

which is $\cos \left(30^{\circ}\right)=\cos \phi(A)=\mu_{1}(A)$. So $\psi_{+}$attains the maximum turning angle $\psi(A)$.

There are other things one could check. But let us do the Euler equation

$$
\frac{A^{2} x}{\left\langle A^{2} x, x\right\rangle}-\frac{2 A x}{\langle A x, x\rangle}+x=0 .
$$

For this we need $\|x\|=1$, so we go back to normalized

$$
\psi_{+}=\frac{3 \sin x-\sin 3 x}{\sqrt{10}} \cdot \frac{1}{\|\sin n x\|}=\frac{1}{\sqrt{5 \pi}}(3 \sin x-\sin 3 x) .
$$

From the above we already know

$$
\begin{aligned}
\left\langle A^{2} \psi_{+}, \psi_{+}\right\rangle & =\left\|A \psi_{+}\right\|^{2}=\frac{45 \pi}{5 \pi}=9, \\
\left\langle A \psi_{+}, \psi_{+}\right\rangle & =\frac{9 \pi}{5 \pi}=\frac{9}{5}
\end{aligned}
$$

and we need

$$
\begin{aligned}
A^{2} \psi_{+} & =-(3 \sin x-9 \sin 3 x)^{\prime \prime} \cdot \frac{1}{\sqrt{5 \pi}} \\
& =(3 \sin x-81 \sin 3 x) \cdot \frac{1}{\sqrt{5 \pi}} .
\end{aligned}
$$

We needed $\left\|\psi_{+}\right\|=1$ in the $\left\langle A^{2} \psi_{+}, \psi_{+}\right\rangle$and $\left\langle A \psi_{+}, \psi_{+}\right\rangle$but a look at the Euler equation which is homogeneous in its numerators means we can now drop the $\sqrt{5 \pi}$ and substitute the other quantities in, from which

$$
\begin{aligned}
& \frac{A^{2} \psi_{+}}{\left\langle A^{2} \psi_{+}, \psi_{+}\right\rangle}-\frac{2 A \psi_{+}}{\left\langle A \psi_{+}, \psi_{+}\right\rangle}+\psi_{+} \\
& \quad=\frac{3 \sin x-81 \sin 3 x}{9}-\frac{2(3 \sin x-9 \sin 3 x)}{9 / 5}+(3 \sin x-\sin 3 x)
\end{aligned}
$$




$$
\begin{aligned}
& =\frac{3 \sin x-81 \sin 3 x-10(3 \sin x-9 \sin 3 x)+27 \sin x-9 \sin 3 x}{9} \\
& =\frac{\sin x[3-30+27]+\sin 3 x[-81+90-9]}{9}=0 .
\end{aligned}
$$

\section{Chapter 6.}

1. I found the unexpected (at least to me) connection to Von Neumann's consulting work at the Aberdeen Proving Grounds (as the Aberdeen laboratories were referred to when I worked at the Naval Research Laboratory in 1959-1963) just by following my curiosity. Therefore, you must follow your own curiosity to uncover historical facets of Ito's work on rocket-flight ballistics in Japan. These days, Google or other search engines will get you instant results. To possibly stoke some further curiosity, I mention that the Ito calculus will figure fundamentally in the financial instruments to which I will apply my operator trigonometry in Chapter 8 of this book.

2. Here is Von Neumann's matrix for $n=3$ :

$$
A=\left[\begin{array}{ccc}
1 & -1 & 0 \\
-1 & 2 & -1 \\
0 & -1 & 1
\end{array}\right]
$$

To find its eigenvalues we set $\operatorname{det}(A-\lambda I)=0$,

$$
0=\operatorname{det}(A-\lambda I)=(1-\lambda)^{2}(2-\lambda)-2(1-\lambda),
$$

from which $\lambda=0,1,3$. Checking this against Von Neumann's general formula $4 \sin ^{2}(k \pi / 2 n)$ for the eigenvalues, we see that the angles therein are $0,30^{\circ}$, and $60^{\circ}$ and thus our $\lambda^{\prime}$ 's do check out.

Generally, the matrices

$$
A=\left[\begin{array}{ccccc}
2 & -1 & 0 & \ldots & 0 \\
-1 & 2 & -1 & \ldots & 0 \\
0 & -1 & 2 & -1 & \vdots \\
\vdots & \ddots & \ddots & \ddots & -1 \\
0 & \ldots & 0 & -1 & 2
\end{array}\right]
$$

are perhaps the most important matrices in numerical linear algebra, as they are the discretization by centered differences at all interior grid 
points for the one-dimensional Laplacian operator $-d^{2} / d x^{2}$. See my book [Gustafson (1999f)]. Von Neumann's statistically inspired matrix is the same except for its first and last rows, which cause the eigenvalue $\lambda=0$ to appear in what otherwise would be a positive definite matrix like that above. Those first and last rows could come, in the PDE context, from Neumann boundary conditions implemented numerically by a first-order centered difference at left and right boundaries of the interval. It does not take much imagination to wonder how other simple ODE or PDE boundary value problems might induce interesting new statistics. That is the direction that seems to me the most interesting in this exercise.

I notice that these eigenvalue $4 \sin ^{2}(k \pi / 2 n)$ also surface in my treatment of numerical stability in my book [Gustafson (1999f), p. 312].

3. Bloomfield and Watson (1975) also used the Lagrange multiplier arguments I gave in Sec. 6.2 to arrive at the inefficiency equation. First they show that the matrices $X^{\prime} \Gamma^{2} X$ and $X^{\prime} \Gamma X$ commute and thus may be diagonalized by the same orthogonal change of basis. Then they find the inefficiency equation in the form

$$
\frac{V x_{i}}{\left\langle V x_{i}, x_{i}\right\rangle}+\frac{V^{-1} x_{i}}{\left\langle V^{-1} x_{i}, x_{i}\right\rangle}=2 x_{i}
$$

Here I have used the more common notation $V$ rather than their $\Gamma$. The $x_{i}$ denotes any column of the optimizing (worst efficiency) regression design matrix $X$. Multiplying this equation by $V$ shows that anything in $\operatorname{sp}\left\{x_{i}, V x_{i}\right\}$ is mapped into that span by $V$. Such two- (or one-) dimensional reducing subspace must have a two- (or one-) dimensional eigenbasis from $V$.

The specific coefficients for the inefficiency vectors and the antieigenvectors are then found from these $2 \times 2$ reduced subsystems as I showed in Sec. 6.2. It might be useful here to clarify two further points one could wonder about when reading their paper.

First is the jump to the quadratic equation

$$
z^{2}-2\langle V x, x\rangle z+\langle V x, x\rangle\left\langle V^{-1} x, x\right\rangle^{-1}=0 .
$$

One need not do that, i.e., one can just by brute force let $x=c_{j} x_{j}+c_{k} x_{k}$ and solve for $c_{j}$ and $c_{k}$ after substituting into the inefficiency equation. See how I did that in the Euler equation in Sec. 3.4. But it is faster to note 
that directly from the inefficiency equation, each of the eigenvalues $\lambda_{j}$ and $\lambda_{k}$ satisfies the scalar quadratic equation.

The second point is the jump from vectors $x$ to matrices $X$ one encounters in this kind of statistical estimation literature. The notation $\left|X^{\prime} A X\right|$ means determinant, but what is meant by $X^{\prime} A X$ itself? For me, it was useful to keep in mind its straightforward linear algebra content. Namely, consider the $2 \times 2$ case. Then write

$$
\begin{aligned}
X^{\prime} A X & =\left[\begin{array}{c}
{\left[x_{1}^{T}\right]} \\
{\left[x_{2}^{T}\right]}
\end{array}\right][A]\left[\left[x_{1}\right]\left[x_{2}\right]\right] \\
& =\left[\begin{array}{c}
{\left[x_{1}^{T}\right]} \\
{\left[x_{2}^{T}\right]}
\end{array}\right]\left[\left[A x_{1}\right]\left[A x_{2}\right]\right] \\
& =\left[\begin{array}{ll}
\left\langle A x_{1}, x_{1}\right\rangle & \left\langle A x_{2}, x_{1}\right\rangle \\
\left\langle A x_{1}, x_{2}\right\rangle & \left\langle A x_{2}, x_{2}\right\rangle
\end{array}\right],
\end{aligned}
$$

which for $A=V$ clearly brings you to the correlation matrix context starting from individual vectors.

4. This is an open-ended research problem for someone other than me. First, one should do a literature search to come up-to-date on any following papers that might have appeared. Is the question obsolete now, from an engineering point of view? Whether that be the case or not, what new pure Rayleigh quotient/Euler equation mathematical theory can be developed, for possible use elsewhere in the future? Hints are the claim of angle bisection in [Cameron (1983)], and that the constraint trace $\left(K K^{T}\right)$ be minimized in [Cameron and Kouvaritakis (1980)]. The latter is an SVD problem, asking for most efficient solution. Indeed the latter paper employs some Moore-Penrose theory. What does the maximization of $\cos ^{2} \theta_{b} \cos ^{2} \theta_{c}$ in [Cameron and Kouvaritakis (1980)] mean operator-theoretically?

5. See my paper [Gustafson (2000d)] where I do this.

6. Given the decomposition $X=M \oplus N$ of the linear space $X$, and $P$ defined by $P x=m$ for each $x=m \oplus n$, one says the $P$ projects on $M$ along $N$. The geometry is that of projecting from the direction of $N$. In the example of the answer to Exercise 3 of Chapter 1, $M$ is $\operatorname{sp}\{x\}$ and $N$ is $\operatorname{sp}\left\{y^{\perp}\right\}$. As to trigonometry, of course $P$ has its internal canonical angle 
theory. I have not pushed an operator trigonometry onto $P$, but an interesting new one might come from its $P=Q R$ factorization, using $R$ in the spirit of my paper [Gustafson (2000d)]. There we noted that for any matrix $A$ factored in both polar and $\mathrm{QR}$ forms, i.e., $A=U|A|=Q R$, one has from $A^{*} A$ that $|R|=|A|$ and hence my entended operator trigonometry applies to $R$ as well. But my suggestion (admittedly vague) here is not that. I suppose I am wondering about the operator trigonometry of upper triangular matrices. One should even create a trigonometry coming just from Jordan canonical forms. How does one bring in the effect of the quasi-nilpotent shift part of $J(\lambda)$ ?

\section{Chapter 7.}

1. We wish to show that any $a, b, c$, all in the interval $[-1,1]$, always satisfy the inequality

$$
a b-b c+a c \leqq 1 .
$$

Here is a proof. From $b^{2} \leqq 1$ and $c^{2} \leqq 1$ we have $b^{2}\left(1-c^{2}\right) \leqq 1-c^{2}$ and hence $b^{2}+c^{2} \leqq 1+b^{2} c^{2}$. Adding $2 b c$ to both sides and multiplying by $a^{2} \leqq 1$ we therefore have $a^{2}\left(b^{2}+c^{2}+2 b c\right) \leqq b^{2}+c^{2}+2 b c \leqq 1+b^{2} c^{2}+2 b c$, that is, $a^{2}(b+c)^{2} \leqq(1+b c)^{2}$. Taking the positive square root yields $a(b+c) \leqq|a||b+c| \leqq 1+b c$.

2. See [Gustafson (2000c)]. Also note the following minor typos in that paper: Theorem 2.2 on p. 38 assumes unit vectors, the second term in the inequality (17) on p. 40 is $1 / 2 \sin ^{2}\left(\theta_{12}\right)$, and on pp. 48 and 49 the factorization referred to is (50), and not (44), which is not a factorization at all.

A simple inequality made equality is just the Cauchy-Schwarz inequality

$$
|\langle u, v\rangle| \leqq\|u\|\|v\|
$$

becoming

$$
|\langle u, v\rangle|=\|u\|\|v\| \cos \theta_{u, v} .
$$

Speaking more generally than just to my new quantum spin inequalities-made-equalities, I would enjoy very much the thought of an accepted legacy that whenever possible, inequalities should be extended to equalities throughout mathematics. Everyone likes to 
prove an inequality to be sharp by exhibiting an example in which equality is obtained. But why not go further and try to fill in all of the nonsharp region with meaningful new terms and thereby replace the full inequality with its inequality-equality?

3. A unitary matrix has its eigenvalues all on the unit circle in the complex plane. While that is great when you want to play with phase, it is defeating when you need differing amplitudes. You have only unit amplitudes for the eigenvalues of a unitary matrix. Stated more simply, we have seen that our operator angles are better thought of as twisting angles rather than just as turning angles.

4. The key is the condition number $\kappa=\lambda_{2} / \lambda_{1}$. One has

$$
\frac{1+\sin \phi(H)}{1-\sin \phi(H)}=\frac{1+\left(\frac{\lambda_{2}-\lambda_{1}}{\lambda_{2}+\lambda_{1}}\right)}{1-\left(\frac{\lambda_{2}-\lambda_{1}}{\lambda_{2}+\lambda_{1}}\right)}=\frac{\lambda_{2}}{\lambda_{1}}=\kappa(H) .
$$

Note that when obtaining these new operator trigonometric identities, one also mixes in some of the customary trigonometry.

5. $M_{\epsilon}=U+\epsilon U^{T}$ has its two eigenvalues

$$
\lambda_{1,2}=\frac{(1+\epsilon) \pm i(1-\epsilon)}{\sqrt{2}}
$$

on the $\left(1+\epsilon^{2}\right)^{1 / 2}$ circle. With $\epsilon>0, M_{\epsilon}$ is still a strongly accretive normal matrix with numerical range $W\left(M_{\epsilon}\right)$ the convex hull of its two eigenvalues. Then, proceeding as in [Gustafson (2000d)], see Example 2 there, computation of the operator angle $\phi_{\mathrm{re}}\left(M_{\epsilon}\right)$ follows from

$$
\cos \phi_{\mathrm{re}}(A)=\mu_{1}(A)=\min \left\{\frac{\beta_{k}}{1\left|\lambda_{k}\right|}\right\},
$$

given the eigenvalues $\lambda_{k}=\beta_{k}+i \delta_{k}$. Thus we have

$$
\phi_{\mathrm{re}}\left(M_{\epsilon}\right)=\cos ^{-1}\left(\frac{1+0.0023}{\sqrt{2}}\right)=44.868^{\circ} .
$$

6. Let us develop the operator trigonometry of $D$. Immediately we find that the operator maximal turning angle and the first antieigenvalue are given by

$$
\mu=\cos \phi(D)=\frac{2 \sqrt{\lambda_{1} \lambda_{2}}}{\lambda_{1}+\lambda_{2}}=(1-r)^{1 / 2}(1+r)^{1 / 2} .
$$

These thus depend only on the distance $r$ from the center of the Bloch sphere. The eigenvectors are easily found and do not depend on $r$. But 
the antieigenvectors do: they are

$$
x^{ \pm}= \pm\left(\frac{1+r}{2}\right)^{1 / 2}\left[\begin{array}{c}
1 \\
e^{i \phi} \tan \frac{\theta}{2}
\end{array}\right]+\left(\frac{1-r}{2}\right)^{1 / 2}\left[\begin{array}{c}
e^{-i \phi} \tan \frac{\theta}{2} \\
-1
\end{array}\right] .
$$

This is reminiscent of a behavior we found in twistors. There the eigenvectors did not depend on the time $t$, whereas the antieigenvectors did depend on $t$. So again the new trigonometry shows that it is the antieigenvectors which are needed to see the "twist" angles of the spin density states.

\section{Chapter 8.}

1. Part (a) follows trivially from the property for $A$ an SPD matrix that

$$
\sin \phi(A)=\frac{\lambda_{1}-\lambda_{n}}{\lambda_{1}+\lambda_{n}}
$$

I note we have used the statistician's ordering of $\lambda_{1}$ as the largest eigenvalue here. As for part (b) we have often used the property that the operator angle $\phi\left(A^{-1}\right)=\phi(A)$. Thus $\cos \phi\left(A^{-1}\right)=\cos \phi(A)$ and $\sin \phi\left(A^{-1}\right)=\sin \phi(A)$, and most of the operator trigonometry of $A^{-1}$ is the same as that of $A$. However, the antieigenvectors of $A^{-1}$ have the same weighting factors as the antieigenvectors of $A$, but the two eigenvectors switch roles. The proof consists of recalling first of all that $A$ and $A^{-1}$ have the same eigenvectors but inverted spectrum, so the eigenvector role-switch is clear. Then check that

$$
\frac{\lambda_{1}\left(A^{-1}\right)}{\lambda_{1}\left(A^{-1}\right)+\lambda_{n}\left(A^{-1}\right)}=\frac{\lambda_{1}(A)}{\lambda_{1}(A)+\lambda_{n}(A)} .
$$

In particular, note that $A$ and $A^{-1}$ have the same operator turning angles, the same eigenvectors, but not the same antieigenvectors.

2. We may equate the expression for $\lambda_{1} /\left(\lambda_{1}+\lambda_{2}\right)$ found in Sec. 8.2 to that found in Lemma 8.4 above. Thus we have

$$
\frac{1+\left[1-\cos ^{2} \phi\left(A_{0}\right) \sin ^{2} \psi(\rho)\right]^{1 / 2}}{2}=\frac{1+\sin \phi(A)}{2}
$$

from which, using $\sin ^{2} \phi(A)+\cos ^{2} \phi(A)=1$, we have Lemma 8.1.

3. Originally, see [Gustafson (2010a)], I was in the enthralls of the entanglements of my operator trigonometry with the financial quantos instrument. But now I see that the trigonometry of Lemma 8.2 just by itself 
is pretty trivial. To show the important (financially) implication of Lemma 8.2,

$$
\rho \sigma_{1} \sigma_{2}\left[\frac{2}{\sigma_{1}^{2}+\sigma_{2}^{2}}\right]=\cos \phi\left(A_{0}\right) \cos \psi(\rho)=\cos \phi(A) \cot \psi(\rho),
$$

just for fun write the last desired equality as

$$
\frac{\cos \phi(A)}{\cos \phi\left(A_{0}\right)}=\frac{\cos \psi(\rho)}{\cot \psi(\rho)} .
$$

The left-hand side by the operator trigonometric cosine formula cancels out to $\sqrt{1-\rho^{2}}$. That is the right-hand side which by elementary trigonometry is just $\sin \psi(\rho)$. To get the first equality, just note that the quantos drift term is

$$
\frac{\rho}{\sqrt{1-\rho^{2}}} \cdot \cos \phi(A)
$$

which is the leftmost term. So it is all about correlations.

Going back to the financial context, do note that our scaling was by the operator trigonometric

$$
\epsilon_{m}=\frac{2}{\sigma_{1}^{2}+\sigma_{2}^{2}}
$$

which gives you the convex minimum

$$
\sin \phi\left(A_{0}\right)=\min _{\epsilon>0}\left\|\epsilon A_{0}-I\right\| .
$$

4. I still like, for practice, to Gauss-reduce small matrices to their inverses. But here the old adjugate formula is much quicker, from which

$$
\Gamma^{-1}=\frac{\left[\begin{array}{cc}
\sigma_{K}^{2} & -\sigma_{2} \sigma_{K} \\
-\sigma_{2} \sigma_{K} & \sigma_{1}^{2}+\sigma_{2}^{2}
\end{array}\right]}{\sigma_{K}^{2} \sigma_{1}^{2}}=\frac{1}{\sigma_{1}^{2}}\left[\begin{array}{cc}
1 & -\frac{\sigma_{2}}{\sigma_{K}} \\
-\frac{\sigma_{1}}{\sigma_{K}} & \frac{\sigma_{1}^{2}+\sigma_{2}^{2}}{\sigma_{K}^{2}}
\end{array}\right] .
$$

Then

$$
\Gamma^{-1}\left[\begin{array}{c}
\sigma_{1} \lambda+\sigma_{2} \lambda_{r} \\
\sigma_{K} \lambda_{r}
\end{array}\right]=\frac{1}{\sigma_{1}^{2}}\left[\begin{array}{c}
\sigma_{1} \lambda \\
-\frac{\sigma_{2} \sigma_{1} \lambda}{\sigma_{K}}+\frac{\sigma_{1}^{2} \lambda_{r}}{\sigma_{K}}
\end{array}\right]=\left[\begin{array}{c}
\frac{\lambda}{\sigma} \\
-\frac{\lambda \sigma_{2}+\lambda_{r} \sigma_{1}}{\sigma_{1} \sigma_{K}}
\end{array}\right] .
$$

5. See the Epilogue in my book [Gustafson (1999f)] and also [Merton (1990)] and especially [Wilmott (1998)].

6. Hint: see American Mathematical Monthly (1968), where I published a paper on compact operators. Thorp also played with those. 


\section{Chapter 9.}

1. $B=\left(S S^{*}\right)^{-1}$ has adjoint

$$
B^{*}=\left(\left(S S^{*}\right)^{-1}\right)^{*}=\left(\left(S S^{*}\right)^{*}\right)^{-1}=\left(S^{* *} S^{*}\right)^{-1}=\left(S S^{*}\right)^{-1}
$$

where we used $S^{* *}=S$ for any closed operator in a Hilbert space.

See [Gustafson (2004b)] for the details about

$$
A^{\prime}=B^{-1} A^{*} B
$$

being $A^{\prime}$ s dual in the new inner product space. It can be tricky and that paper reveals a serious error in some preceding papers in the numerical linear algebra literature.

By the way, since as shown in Sec. 9.1 we have

$$
\langle A x, x\rangle_{B}=\langle D y, y\rangle, \quad y=S^{-1} x,
$$

you may check that similarly we have

$$
\frac{\left|\langle A x, x\rangle_{B}\right|}{\|A x\|_{B}\|x\|_{B}}=\frac{|\langle D y, y\rangle|}{\|D y\|\|y\|} .
$$

Thus the total antieigenvalue theory for $A$ in the $B$-inner product is essentially (up to antieigenvectors) that of $D$ in the original space.

2. $U$ is not normal. However its eigenvalues and eigenvectors are easily calculated and one arrives at its diagonal representation

$$
A=\left[\begin{array}{cccc}
-\frac{1}{2}+i \frac{\sqrt{3}}{2} & 0 & 0 & 0 \\
0 & -\frac{1}{2}-i \frac{\sqrt{3}}{2} & 0 & 0 \\
0 & 0 & 1 & 0 \\
0 & 0 & 0 & 0
\end{array}\right] .
$$

What I found equally interesting in [Gustafson (2011a)] was that $A^{\prime}$ s normal polynomial, that is, the polynomial $n(\lambda)$ such that $A^{*}$ is represented as $A^{*}=n(A)$, is also informationally deficient: $A^{*}=A^{2}$. Usually, in fact almost always, one finds $A^{*}=n(A)$ where $n(\lambda)$ has degree $n-1$ for a given $n \times n$ matrix $A$. Surprisingly, the loss of terms in $n(\lambda)$ can be related to gravitational lensing. This phenomenon will be treated in a paper, Gustafson, Normal Degree Revisited, to appear. 
3. This is fun. We can also do some variations. For $x=x_{1}+x_{n}$ and $y=x_{1}-x_{n}$ we obtain straightforwardly

$$
\frac{\langle A x, A y\rangle}{\|A x\|\|A y\|}=\frac{\lambda_{1}^{2}-\lambda_{n}^{2}}{\lambda_{1}^{2}+\lambda_{n}^{2}} .
$$

The right-hand side is $\sin \phi\left(A^{2}\right)$. However, we may for the moment ignore that and just notice that from conventional trigonometric identities, we have from the definition of the $\mathrm{K}-\mathrm{W}$ angle from the conventional condition number $\kappa$ according to

$$
\cot \left(\frac{\theta(A)}{2}\right)=\kappa
$$

that

$$
\cos \theta(A)=\frac{\kappa^{2}-1}{\kappa^{2}+1}=\frac{\lambda_{n}^{2}-\lambda_{1}^{2}}{\lambda_{1}^{2}+\lambda_{1}^{2}} .
$$

Thus $x=x_{1}+x_{n}$ and $y=x_{1}+x_{n}$ are optimal. They can of course be made of norm one due to the homogeneity of the $\mathrm{K}-\mathrm{W}$ inequality entities.

Let us show here a variation employing instead the general operator trigonometric identity (see Lemma 4.1 in Sec. 4.3)

$$
\sin \phi\left(A^{1 / 2}\right)=\frac{\sin \phi(A)}{1+\cos \phi(A)} .
$$

We have, continuing on from the above,

$$
\begin{aligned}
\frac{\langle A x, A y\rangle}{\|A x\|\|A y\|} & =\frac{\lambda_{1}^{2}-\lambda_{n}^{2}}{\lambda_{1}^{2}+\lambda_{n}^{2}}=\left(\frac{\lambda_{1}-\lambda_{n}}{\lambda_{1}+\lambda_{n}}\right) \frac{\left(\lambda_{1}+\lambda_{n}\right)^{2}}{\left(\lambda_{1}^{2}+\lambda_{n}^{2}\right)} \\
& =\sin \phi(A)\left[1+\frac{2 \lambda_{1} \lambda_{n}}{\lambda_{1}^{2}+\lambda_{n}^{2}}\right] \\
& =\sin \phi(A)\left[1+\cos \phi\left(A^{2}\right)\right] \\
& =\frac{\sin \phi\left(A^{2}\right)}{\left[1+\cos \phi\left(A^{2}\right)\right]} \cdot\left[1+\cos \phi\left(A^{2}\right)\right]
\end{aligned}
$$

and we are back again to the result that the $\mathrm{K}-\mathrm{W}$ inequality is optimized to either $\cos \theta(A)$ from the original Kantorovich-Wielandt standard condition number view, or at $\sin \phi\left(A^{2}\right)$ from my view. The merit in this instance of the latter viewpoint is my point in Sec. 9.3 that 
my $\sin \phi(A)$ convex minimum, applied in this case to the $\sin \phi\left(A^{2}\right)$ minimum, contains the Kantorovich-Wielandt sharp vectors $x=x_{1}+x_{n}$ and $y=x_{1}-x_{n}$.

4. We may consider $x_{+}$, the case of $x_{-}$following in the same way. For convenience write

$$
x_{+}=\frac{\lambda_{1}^{1 / 4} x_{1}+\lambda_{n}^{1 / 4} x_{n}}{d}, \quad d=\left(\lambda_{1}^{1 / 2}+\lambda_{n}^{1 / 2}\right) .
$$

Then

$$
\langle A x, x\rangle-\left\langle A^{-1} x, x\right\rangle^{-1}=\frac{\lambda_{1}^{3 / 2}+\lambda_{n}^{3 / 2}}{d^{2}}-\frac{d^{2}}{\lambda_{1}^{-1 / 2}+\lambda_{n}^{-1 / 2}} .
$$

Using the factorization $(a+b)^{3}=(a+b)\left(a^{2}-a b+b^{2}\right)$, the first term is $\lambda_{1}-\lambda_{1}^{1 / 2} \lambda_{n}^{1 / 2}+\lambda_{n}$. Thus we need another $\lambda_{1}^{1 / 2} \lambda_{n}^{1 / 2}$ from the second term, which follows by elementary algebra.

The fact that these optimal $x_{ \pm}$are the antieigenvectors for the operator $A^{-1 / 2}$ means that maximizing the Shisha-Mond expression is equivalent to minimizing the expression

$$
\frac{\left\langle A^{-1 / 2} x, x\right\rangle}{\left\|A^{-1 / 2} x\right\|\|x\|} .
$$

It would be nice to work out the full details relating the two theories.

5. Writing the detail in vector component form, we have

$$
\begin{aligned}
x_{+} & =\left(\lambda_{n}^{1 / 2}, 0, \ldots, 0, \lambda_{1}^{1 / 2}\right)\left(\lambda_{1}+\lambda_{n}\right)^{-1 / 2}, \\
A x_{+} & =\left(\lambda_{n}^{1 / 2} \lambda_{1}, 0, \ldots, 0, \lambda_{1}^{1 / 2} \lambda_{n}\right)\left(\lambda_{1}+\lambda_{n}\right)^{-1 / 2}
\end{aligned}
$$

and we recall that $\left\|A x_{ \pm}\right\|=\lambda_{n}^{1 / 2} \lambda_{1}^{1 / 2}$. For $y=A x_{+}$the slack variable numerator of the expression for $\sin \phi(A)$ in Theorem 9.1 is, writing everything out in that $i j$ sum to be absolutely clear,

$$
\begin{aligned}
& \left(x_{1} y_{2}-y_{1} x_{2}\right)^{2}+\left(x_{1} y_{3}-y_{1} x_{3}^{2}\right)+\cdots+\left(x_{1} y_{n}-y_{1} x_{n}\right)^{2} \\
& \quad+\left(x_{2} y_{3}-y_{2} x_{3}\right)^{2}+\cdots+\left(x_{2} y_{n}-y_{2} x_{n}\right)^{2} \cdots \\
& \quad+\left(x_{n-1} y_{n}-y_{n-1} x_{n}\right)^{2} \\
& =(O)^{2}+(O)^{2}+\cdots+\left(\lambda_{n}^{1 / 2} \lambda_{1}^{1 / 2} \lambda_{n}-\lambda_{n}^{1 / 2} \lambda_{1} \lambda_{1}^{1 / 2}\right)^{2} \cdot\left(\lambda_{1}+\lambda_{n}\right)^{-2} \\
& \quad+(O)^{2}+\cdots+\cdots+(O)^{2} \cdots+(O)^{2} .
\end{aligned}
$$


6. (a) The first antieigenvalue is

$$
\mu_{1}=\cos \phi(A)=\frac{2 \lambda^{2}}{\lambda^{4}+1}=\frac{2(1+\lambda)}{(2+3 \lambda)+1}=\frac{2}{3} .
$$

Here I found it fun to play with the golden mean $\lambda$, from which one stumbles into the reductions

$$
\lambda^{2}=1+\lambda, \quad \lambda^{4}=2+3 \lambda, \quad \lambda^{6}=5+8 \lambda, \quad \lambda^{3}=1+2 \lambda .
$$

Hence $A^{\prime}$ s operator angle is $\phi(A) \cong 48.1896851^{\circ}$. Also we have independently

$$
\nu_{1}=\sin \phi(A)=\frac{\lambda^{4}-1}{\lambda^{4}+1}=\frac{3 \lambda+1}{3 \lambda+3}=\frac{1}{3}+\frac{2}{3}\left(\frac{1}{\lambda}\right) .
$$

Thus we may check that $\sin ^{2} \phi(A)+\cos ^{2} \phi(A)=1$ by checking

$$
\left(\frac{1}{3}+\frac{2}{3}\left(\frac{1}{\lambda}\right)\right)^{2}+\frac{4}{9}=\frac{1}{9}\left[5+4\left(\frac{1}{\lambda^{2}}+\frac{1}{\lambda}\right)\right]=\frac{9}{9} .
$$

Next we may form the antieigenvectors

$$
x_{ \pm}=\left(\frac{\lambda^{2}}{\sqrt{\lambda^{4}+1}}\right) \cdot \frac{1}{\sqrt{\lambda^{2}+1}}\left[\begin{array}{c}
-1 \\
\lambda
\end{array}\right] \pm\left(\frac{1}{\sqrt{\lambda^{4}+1}}\right) \cdot \frac{1}{\sqrt{\lambda^{2}}+1}\left[\begin{array}{c}
\lambda \\
1
\end{array}\right] .
$$

Let

$$
\begin{aligned}
d & =\sqrt{\lambda^{4}+1} \cdot \sqrt{\lambda^{2}+1}=\left(\lambda^{6}+\lambda^{4}+\lambda^{2}+1\right)^{1 / 2} \\
& =(12 \lambda+9)^{1 / 2} \cong 5.330704256 .
\end{aligned}
$$

Then

$$
\begin{aligned}
& x_{+}=\frac{1}{d}\left[\begin{array}{c}
-\lambda^{2}+\lambda \\
\lambda^{3}+1
\end{array}\right]=\frac{1}{d}\left[\begin{array}{c}
-1 \\
2 \lambda+2
\end{array}\right] \cong\left[\begin{array}{c}
-0.1876 \\
0.9824
\end{array}\right], \\
& x_{-}=\frac{1}{d}\left[\begin{array}{c}
-\lambda^{2}-\lambda \\
\lambda^{3}-1
\end{array}\right]=\frac{1}{d}\left[\begin{array}{c}
-1-2 \lambda \\
2 \lambda
\end{array}\right] \cong\left[\begin{array}{c}
-0.7948 \\
0.3686
\end{array}\right] .
\end{aligned}
$$

Notice

$$
\left\|x_{+}\right\|^{2}=\frac{1+4 \lambda^{2}+4+8 \lambda}{12 \lambda+9}=\frac{12 \lambda+9}{12 \lambda+9}=1 .
$$

Similarly, as a check, we have

$$
\left\|x_{-}\right\|^{2}=\frac{1+4 \lambda+8 \lambda^{2}}{12 \lambda+9}=1 .
$$


(b) In the $A$-inner product we have

$$
\left\langle x_{+}, x_{-}\right\rangle_{A} \equiv\left\langle A x_{+}, x_{-}\right\rangle=\frac{\lambda_{n} \lambda_{1}}{\lambda_{1}+\lambda_{n}}\left[\left\langle x_{1}, x_{1}\right\rangle-\left\langle x_{n}, x_{n}\right\rangle\right]=0 .
$$

So the full antieigenvector set $\left\{x_{ \pm}\right\}$becomes an orthonormal basis in the $A$-inner product.

(c) We may compute the $A$ inner product

$$
\begin{aligned}
\left\langle A x_{+}, x_{-}\right\rangle & =\frac{\left\langle\left[\begin{array}{ll}
2 & 1 \\
1 & 1
\end{array}\right]\left[\begin{array}{c}
-1 \\
2 \lambda+1
\end{array}\right],\left[\begin{array}{c}
-1-2 \lambda \\
2 \lambda
\end{array}\right]\right\rangle}{(12 \lambda+9)} \\
& =\frac{[-1-2 \lambda, 2 \lambda]}{(12 \lambda+9)}\left[\begin{array}{c}
2 \lambda \\
2 \lambda+1
\end{array}\right] \\
& =\frac{-2 \lambda-4 \lambda^{2}+4 \lambda^{2}+2 \lambda}{12 \lambda+9}=0 .
\end{aligned}
$$

In the original inner product we have

$$
\begin{aligned}
\left\langle x_{+}, x_{-}\right\rangle & =\frac{(1+2 \lambda)+(2 \lambda+2)(2 \lambda)}{(12 \lambda+9)} \\
& =\frac{1+6 \lambda+4 \lambda^{2}}{12 \lambda+9}=\frac{10 \lambda+5}{12 \lambda+9} \\
& =\frac{10+5 \sqrt{5}}{15+6 \sqrt{5}}=\frac{21.18033989}{28.41640787} \\
& =0.74535599=\sin \phi(A) .
\end{aligned}
$$




\section{Bibliography}

Bib. 1 lists the author's contributions (sometimes with coauthors) which have operator-trigonometric content. Bib. 2 lists what he deems important contributions by others which have direct, or indirect, operatortrigonometric content. Bib. 3 lists the remaining bibliography which were cited in this book.

\section{Bib. 1 Contributions by K. Gustafson (and Coauthors)}

K. Gustafson. (1966). A Perturbation Lemma, Bull. Amer. Math. Soc. 72, pp. 334-338.

K. Gustafson. (1967). Positive Operator Products, Notices Amer. Math. Soc. 14, Abstract 67T-531, p. 717. See also Abstracts 67T-340, 67T-564, 67T-675.

K. Gustafson. (1968a). A Note on Left Multiplication of Semi-group Generators, Pacific J. Math. 24, pp. 463-465.

K. Gustafson. (1968b). The Angle of an Operator and Positive Operator Products, Bull. Amer. Math. Soc. 74, pp. 488-492.

K. Gustafson. (1968c). Positive (noncommuting) Operator Products and Semigroups, Math. Zeitschrift 105, pp. 160-172.

K. Gustafson. (1968d). A Min-Max Theorem, Amer. Math. Soc. Notices 15, p. 799.

K. Gustafson. (1969a). Doubling Perturbation Sizes and Preservation of Operator Indices in Normed Linear Spaces, Proc. Camb. Phil. Soc. 98, pp. 281-294.

K. Gustafson. (1969b). On the Cosine of Unbounded Operators, Acta Sci. Math. 30, pp. 33-34 (with B. Zwahlen).

K. Gustafson. (1969c). Some Perturbation Theorems for Nonnegative Contraction Semi-Groups, J. Math. Soc. Japan 21, pp. 200-204 (with Ken-iti Sato).

K. Gustafson. (1970a). A Simple Proof of the Toeplitz-Hausdorff Theorem for Linear Operators, Proc. Amer. Math. Soc. 25, pp. 203-204.

K. Gustafson. (1972a). Anti-eigenvalue Inequalities in Operator Theory, Inequalities III, Shisha, O. (ed.), (Academic Press), pp. 115-119. 
K. Gustafson. (1972b). Multiplicative Perturbation of Semigroup Generators, Pac. J. Math. 41, pp. 731-742 (with G. Lumer).

K. Gustafson. (1972c). Multiplicative Perturbation of Nonlinear m-Accretive Operators, J. Funct. Anal. 10, pp. 149-158 (with B. Calvert).

K. Gustafson. (1977a). Numerical Range and Accretivity of Operator Products, J. Math. Anal. Applic. 60, pp. 693-702 (with D. Rao).

K. Gustafson. (1983a). The RKNG (Rellich, Kato, Nagy, Gustafson) Perturbation Theorem for Linear Operators in Hilbert and Banach Space, Acta Sci. Math. 45, pp. 201-211.

K. Gustafson. (1989a). Antieigenvalue Bounds, J. Math. Anal. Applic. 143, pp. 327-340 (with M. Seddighin).

K. Gustafson. (1991a). Antieigenvalues in Analysis, in Fourth International Workshop in Analysis and its Applications, Stanojevic, C. and Hadzic, O. (eds.), Dubrovnik, Yugoslavia, June 1-10, 1990 (Novi Sad, Yugoslavia), pp. 57-69.

K. Gustafson. (1993a). A Note on Total Antieigenvectors, J. Math. Anal. Applic. 178, pp. 603-611 (with M. Seddighin).

K. Gustafson. (1994a). Operator Trigonometry, Linear and Multilinear Algebra 37, pp. 139-159.

K. Gustafson. (1994b). Antieigenvalues, Lin. Alg. and Applic. 208/209, pp. 437-454.

K. Gustafson. (1994c). Computational Trigonometry, Proc. Colorado Conf. on Iterative Methods, Vol. 1, p. 1.

K. Gustafson. (1995a). Matrix Trigonometry, Lin. Alg. and Applic. 217, pp. 117-140.

K. Gustafson. (1996a). Lectures on Computational Fluid Dynamics, Mathematical Physics, and Linear Algebra (Kaigai Publishers, Tokyo, Japan).

K. Gustafson. (1996b). Trigonometric Interpretation of Iterative Methods, in Proc. Conf. Algebraic Multilevel Iteration Methods with Applications, Axelsson, O. and Polman, B. (eds.), (Nijmegen, Netherlands), June 13-15, pp. 23-29.

K. Gustafson. (1996c). Commentary on Topics in the Analytic Theory of Matrices, Section 23, Singular Angles of a Square Matrix, in Collected Works of Helmut Wielandt 2, Huppert, B. and Schneider, H. (eds.), (De Gruyters, Berlin), pp. 356-367.

K. Gustafson. (1997a). Numerical Range: The Field of Values of Linear Operators and Matrices (Springer, Berlin), (with D. Rao).

K. Gustafson. (1997b). Operator Trigonometry of Iterative Methods, Num. Lin. Alg. with Applic. 34, pp. 333-347.

K. Gustafson. (1997c). Antieigenvalues, Encyclopaedia of Mathematics, Supplement 1, (Kluwer Acad. Publ., Dordrecht), p. 57.

K. Gustafson. (1997d). Lectures on Computational Fluid Dynamics, Mathematical Physics, and Linear Algebra, (World Scientific, Singapore).

K. Gustafson. (1998a). Domain Decomposition, Operator Trigonometry, Robin Condition, Contemp. Math. 218, pp. 455-460. 
K. Gustafson. (1998b). Operator Trigonometry of Wavelet Frames, in Iterative Methods in Scientific Computation, Wang, J., Allen, M., Chen, B., Mathew, T. (eds.), IMACS Series in Computational and Applied Mathematics, Vol. 4 (New Brunswick, NJ), pp. 161-166.

K. Gustafson. (1998c). Semigroups and Antieigenvalues, in Irreversibility and Causality - Semigroups and Rigged Hilbert Spaces, Bohm, A., Doebner, H. and Kielanowski, P. (eds.), Lecture Notes in Physics, Vol. 504, (Springer, Berlin), pp. 379-384.

K. Gustafson. (1998d). Operator Trigonometry of Linear Systems, in Proc. 8th IFAC Symposium on Large Scale Systems: Theory and Applications, Koussoulas, N. and Groumpos, P. (eds.), Patras, Greece, July 15-17, (Pergamon Press, 1999), pp. 950-955.

K. Gustafson. (1998e). Symmetrized Product Definiteness? Comments on Solutions 19-5.1-19-5.5, IMAGE: Bull. Int. Linear Algebra Soc. 21, p. 22.

K. Gustafson. (1998f). Antieigenvalues: An Extended Spectral Theory, in Generalized Functions, Operator Theory and Dynamical Systems, Antoniou, I. and Lumer, G. (eds.), Pitman Research Notes in Mathematics, Vol. 399, London, pp. 144-149.

K. Gustafson. (1998g). Operator Trigonometry of the Model Problem, Num. Lin. Alg. with Applic. 5, pp. 377-399.

K. Gustafson. (1999a). The Geometry of Quantum Probabilities, in On Quanta, Mind, and Matter: Hans Primas in Context, Atmanspacher, H., Amann, A., and MuellerHerold, U. (eds.), (Kluwer, Dordrecht), pp. 151-164.

K. Gustafson. (1999b). The Geometrical Meaning of the Kantorovich-Wielandt Inequalities, Lin. Alg. and Applic. 296, pp. 143-151.

K. Gustafson. (1999c). Symmetrized Product Definiteness: A Further Comment, IMAGE: Bull. Int. Linear Algebra Soc. 22, p. 26.

K. Gustafson. (1999d). A Computational Trigonometry and Related Contributions by Russians Kantorovich, Krein, Kaporin, Computational Technologies 4(3), pp. 73-83, (Novosibirsk, Russia).

K. Gustafson. (1999e). On Geometry of Statistical Efficiency, preprint.

K. Gustafson. (2000a). The Trigonometry of Quantum Probabilities, in Trends in Contemporary Infinite Dimensional Analysis and Quantum Probability, Accardi, L., Kuo, H., Obata, N., Saito, K., Si, S., and Streit, L. (eds.), (Italian Institute of Culture, Kyoto), pp. 159-173.

K. Gustafson. (2000b). Semigroup Theory and Operator Trigonometry, in Semigroups of Operators: Theory and Applications, Balakrishnan, A.V. (ed.), (Birkhäuser, Basel), pp. 131-140.

K. Gustafson. (2000c). Quantum Trigonometry, Inf. Dim. Anal. Quant. Probab. Relat. Top. 3, pp. 33-52.

K. Gustafson. (2000d). An Extended Operator Trigonometry, Lin. Alg. and Applic. 319, pp. 117-135. 
K. Gustafson. (2001a). An Unconventional Linear Algebra: Operator Trigonometry, in Unconventional Models of Computation, UMC'2K, Antoniou, I., Calude, C., and Dinneen, M. (eds.), (Springer, London), pp. 48-67.

K. Gustafson. (2001b). Probability, Geometry, and Irreversibility in Quantum Mechanics, Chaos, Solitons and Fractals 12, pp. 2849-2858.

K. Gustafson. (2002a). Operator Trigonometry of Statistics and Econometrics, Lin. Alg. and Applic. 354, pp. 151-158.

K. Gustafson. (2002b). CP-Violation as Antieigenvector-Breaking, Adv. Chem. Phys. 122, pp. 239-258.

K. Gustafson. (2003a). Operator Trigonometry of Preconditioning, Domain Decomposition, Sparse Approximate Inverses, Successive Overrelaxation, Minimum Residual Schemes, Num. Lin. Alg. with Applic. 10, pp. 291-315.

K. Gustafson. (2003b). Bell's Inequalities, in Contributions to the XXII Solvay Conference on Physics, Borisov, A. (ed.), (Moscow-Izhevsk, ISC, Moscow State University), pp. 501-517.

K. Gustafson. (2003c). Bell's Inequality and the Accardi-Gustafson Inequality, in Foundations of Probability and Physics-2, Khrennikov, A. (ed.), (Växjo University Press, Sweden), pp. 207-223.

K. Gustafson. (2003d). Bell's Inequalities, in The Physics of Communication, Proceedings of the XXII Solvay Conference on Physics, Antoniou, I., Sadovnichy, V., and Walther, H. (eds.), (World Scientific), pp. 534-554.

K. Gustafson. (2003e). in Preconditioning, Inner Products, Normal Degree, 2003 International Conference on Preconditioning Techniques for Large Sparse Matrix Problems in Scientific and Industrial Applications, Ng, E., Saad, Y., and Tang, W. P. (eds.), (NAPA, CA, October), 3 pp.

K. Gustafson. (2004a). An Inner Product Lemma, Num. Lin. Alg. and Applic. 11, pp. 649-659.

K. Gustafson. (2004b). Normal Degree, Num. Lin. Alg. and Applic. 11, pp. 661-674.

K. Gustafson. (2004c). Interaction Antieigenvalues, J. Math. Anal. Applic. 299, pp. 174-185.

K. Gustafson. (2005a). The Geometry of Statistical Efficiency, Res. Lett. Inf. Math. Sci. 8, pp. 105-121.

K. Gustafson. (2005b). On the Eigenvalues which Express Antieigenvalues, Int. J. Math. Math. Sci. 10, pp. 1543-1554 (with M. Seddighin).

K. Gustafson. (2005c). Bell and Zeno, Int. J. Theor. Phys. 44, pp. 1931-2940.

K. Gustafson. (2006a). Noncommutative Trigonometry, in Operator Theory: Advances and Applications 167, pp. 127-155.

K. Gustafson. (2006b). The Trigonometry of Matrix Statistics, Int. Statist. Rev. 74, pp. 187-202.

K. Gustafson. (2007a). Noncommutative Trigonometry and Quantum Mechanics, in Advances in Deterministic and Stochastic Analysis, Chuong N., Ciarlet P., Lax P., Mumford D. and Phong D. (eds.), (World Scientific), pp. 341-360. 
K. Gustafson. (2007b). The Geometry of Statistical Efficiency and Matrix Statistics, J. Appl. Math. Decis. Sci., doi:10.1155/2007/94515.

K. Gustafson. (2008a). The Operator Trigonometry in Statistics, in Functional and Operatorial Statistics, Dabo-Niang S. and Ferraty F. (eds.), pp. 189-193.

K. Gustafson. (2009a). The Trigonometry of Twistors and Elementary Particles, in Foundations of Probability and Physics-5, Accardi L., Adenier G., Fuchs C. A., Jaeger G., Khrennikov A. Yu., Larsson, J. A. and Stenholm S. (eds.), Amer. Inst. of Physics Conference Proceedings, Vol. 1101(AIP), pp. 65-73.

K. Gustafson. (2009b). Operator Trigonometry of Hotelling Correlation, Frobenius Condition, Penrose Twistor, Lin. Alg. and Applic. 430, pp. 2762-2770.

K. Gustafson. (2010a). Operator Trigonometry of Multivariate Finance, J. Multivar. Anal. 101, pp. 374-384.

K. Gustafson. (2010b). Slant Antieigenvalues and Slant Antieigenvectors, Lin. Alg. and Applic. 432, pp. 1348-1362 (with M. Seddighin).

K. Gustafson. (2010c). A Trigonometry of Quantum States, in Quantum Theory: Reconsideration of Foundations-5, Khrennikov A. (ed.), Amer. Inst. of Physics Conference Proceedings, Vol. 1232(AIP), pp. 72-85.

K. Gustafson. (2010d). On my Min-Max Theorem (1968) and its Consequences, Acta Comment. Univ. Tartu. Math. 14, pp. 45-51.

K. Gustafson. (2010e). Operator Geometry of Statistics, The Oxford Handbook of Functional Data Analysis, Ferraty F. and Romain Y. (eds.), (Oxford University Press), pp. 355-382.

K. Gustafson. (2011a). Trigonometry of Quantum States, Found. Phys. 41, 450-465.

K. Gustafson. (2011b). Forty Years of Antieigenvalue Theory and Applications, Lecture given at IWMS 2010, Shanghai, China, preprint.

K. Gustafson. (2011c). Geometric Invariants and Operator Trigonometry, in preparation.

K. Gustafson. (2012a). The Financial Sharpe Ratio Seen as a New Risk Ratio via Antieigenvalue Theory, in preparation.

\section{Bib. 2 Important Contributions by Others}

L. Accardi and A. Fedullo. (1982). On the Statistical Meaning of Complex Numbers in Quantum Mechanics, Lett. Nuovo. Cim. 34, pp. 161-172.

E. Asplund and V. Ptak. (1971). A Minmax Inequality for Operators and a Related Numerical Range, Acta Math. 126, pp. 53-62.

C. Davis. (1980). Extending the Kantorovich Inequalities to Normal Matrices, Lin. Alg. and Appl. 31, pp. 173-177.

S. Drury, S. Liu, C. Y. Lu, S. Puntanen and G. P. H. Styan. (2002). Some Comments on Several Matrix Inequalities with Applications to Canonical Correlations: Historical Background and Recent Developments, Sankhya 64, Series A, Pt. 2, pp. 453-507. 
P. Hess. (1971). A Remark on the Cosine of Linear Operators, Acta Sci. Math. 32, pp. 267-269.

S. Hossein, K. Paul, L. Debnath and K. Das. (2008). Symmetric Antieigenvalue and Symmetric Antieigenvector, J. Math. Anal. Applic. 345, pp. 771-776.

L. Kantorovich. (1948). Functional Analysis and Applied Mathematics, Uspekhi Mat. Nauk 3(6), pp. 89-185.

R. Khatree. (2001). On Calculation of Antieigenvalues and Antieigenvectors, J. Interdiscip. Math. 4, pp. 195-199.

R. Khatree. (2002). On Generalized Antieigenvalue and Antieigenmatrix of order $r$, Amer. J. of Math. Management Sci. 22, pp. 89-98.

R. Khatree. (2003). Antieigenvalues and Antieigenvectors in Statistics, J. Statist. Plan. Inference 114, pp. 131-144.

M. G. Krein. (1969). Angular Localization of the Spectrum of a Multiplicative Integral in a Hilbert Space, Funct. Anal. Appl. 3, pp. 89-90.

B. A. Mirman. (1983). Antieigenvalues: Method of Estimation and Calculation, Lin. Alg. and Appl. 49, pp. 247-255.

C. R. Rao. (2005). Antieigenvalues and Antisingularvalues of a Matrix and Application to Problems in Statistics, Res. Lett. Inf. Math. Sci. 8, pp. 53-76.

C. R. Rao. (2007). Antieigenvalues and Antisingularvalues of a Matrix and Application to Problems in Statistics, Math. Inequal. Appl. 10, pp. 471-489.

D. Rao. (1972). Numerical Range and Positivity of Operator Products, Ph.D. Dissertation, University of Colorado, Boulder, Colorado.

M. Seddighin. (2002). Antieigenvalues and Total Antieigenvalues of Normal Operators, J. Math. Anal. Appl. 274, pp. 239-254.

M. Seddighin. (2005). On the Joint Antieigenvalue of Operators on Normal Subalgebras, J. Math. Anal. Applic. 312, pp. 61-71.

M. Seddighin. (2009). Antieigenvalue Techniques in Statistics, Lin. Alg. and Applic. 430, pp. 2566-2580.

M. Seddighin. (2011). Slant Joint Antieigenvalues and Antieigenvectors of Operators in Normal Subalgebras, Lin. Alg. and Applic. 434, pp. 1395-1408.

H. Wielandt. (1967). Topics in the Analytic Theory of Matrices, University of Wisconsin Lecture Notes, Madison, Wisconsin.

\section{Bib. 3 Other General and Specific Cited References}

A. Afriat and F. Seleri. (1999). The Einstein, Podolski, and Rosen Paradox, (Plenum Press, New York).

J. Andreason, B. Jensen and R. Poulsen. (1998). Eight Valuation Methods in Financial Mathematics: The Black-Scholes Formula as an Example, Math. Scientist 23, pp. 18-40.

A. Aspect, J. Dalibard and G. Roger. (1982). Experimental Test of Bell's Inequalities Using Time-Varying Analyzers, Phys. Rev. Lett. 49, pp. 1804-1807. 
J. Authers. (2010). The Fearful Rise of Markets, (Financial Times Press).

O. Axelsson. (1994). Iterative Solution Methods, (Cambridge Univ. Press).

I. Bajeux-Besnainou and R. Portait. (2002). Dynamic, Deterministic and Static Optimal Portfolio Strategies in a Mean-Variance Framework Under Stochastic Interest Rates, in Wilmott P. and Rasmussen H. (eds.), New Directions in Mathematical Finance, (John Wiley \& Sons Ltd., London), pp. 101-115.

M. Baxter and A. Rennie. (1996). Financial Calculus, (Cambridge University Press, Cambridge, UK).

J. Bell. (1964). On the Einstein-Podolsky-Rosen Paradox, Physics 1, pp. 195-200.

I. Bengtsson and K. Zyczkowski. (2008). Geometry of Quantum States, (Cambridge Univ. Press, Cambridge).

J. Bernasconi and K. Gustafson. (1992). Human and Machine "Quick Modelling". Adv. Neural Info. Process. Syst. 4, pp. 1151-1158.

J. Bernasconi and K. Gustafson. (1994). Inductive Inference and Neural Nets. Network: Comput. Neural Syst. 5, pp. 203-228.

J. Bernasconi and K. Gustafson. (1998). Contextual Quick-Learning and Generalization by Humans and Machines. Network: Comput. Neural Syst. 9, pp. 85-106.

P. Bloomfield and G. S. Watson. (1975). The Inefficiency of Least Squares, Biometrika 62, pp. 121-128.

U. Brehm. (1990). The Shape Invariant of Triangles and Trigonometry in Two-Point Homogeneous Spaces, Geom. Dedicata 33, pp. 59-76.

R. Cameron and B. Kouvaritakis. (1980). Minimizing the Norm of Output Feedback Controllers Used in Pole Placement: A Dyadic Approach, Int. J. Control 32, pp. 759-770.

R. Cameron. (1983). Minimizing the Product of Two Rayleigh Quotients, Linear and Multilinear Algebra 13, pp. 177-778.

R. Carmona and V. Durrleman. (2003). Pricing and Hedging Spread Options, SIAM Review 45, pp. 627-685.

P. Carr, H. Geman and D. Madan. (2001). Pricing and Hedging in Incomplete Markets, J. Financial Economics 62, pp. 131-167.

F. Chaitin-Chatelin and S. Gratton. (2000). On the Condition Numbers Associated with the Polar Factorization of a Matrix, Numer. Lin. Alg. Appl. 7, pp. 337-354.

K. L. Chu, J. Isotalo, S. Puntanen, and G. P. H. Styan. (2005). The Efficiency Factorization Multiplier for the Watson Efficiency in Partitioned Linear Models: Some Examples and a Literature Review, Res. Lett. Inf. Math. Sci. 8, pp. 165-187.

C. Chui. (1992). An Introduction to Wavelets, (Academic Press).

J. Clauser, M. Horne, A. Shimony and R. Holt. (1969). Proposed Experiment to Test Local Hidden-Variable Theories, Phys. Rev. Lett. 23, pp. 880-884.

Y. Coudene. (2006). Pictures of Hyperbolic Dynamical Systems, Notices of the AMS 53(1), pp. 8-13.

D. Cudia. (1964). The Geometry of Banach Spaces. Smoothness, Trans. Amer. Math. Soc. 110, pp. 284-314. 
N. Cuntoor and R. Chellappa. (2006). Key Frame-Based Activity Representation Using Antieigenvalues, in Computer Vision-ACCV 2006, Narayanan P., Nayar S., and Shum H. Y. (eds.), Springer Lecture Notes in Computer Science, Vol. 3852/2006, (Springer), pp. 499-508.

I. Daubechies. (1990). The Wavelet Transform, Time-Frequency Localization and Signal Analysis, IEEE Trans. Information Theory 36, pp. 961-1005.

I. Daubechies. (1992). Ten Lectures on Wavelets, (SIAM Publications, Philadelphia).

J. Dauxois, G. Nkiet and Y. Romain. (2004). Canonical Analysis Relative to a Closed Subspace, Lin. Alg. in Applic. 388, pp. 119-145.

F. Delbaen and W. Schachermayer. (2006). The Mathematics of Arbitrage, (Springer, Berlin).

J. Dorroh. (1966). Contraction Semigroups in a Function Space, Pacific J. Math. 19, pp. 35-38.

R. Duffin and A. Schaeffer. (1952). A Class of Nonharmonic Fourier Series, Trans. Amer. Math. Soc. 72, pp. 341-366.

N. Dunford and J. Schwartz. (1971). Linear Operators III: Spectral Operators, (Wiley \& Sons, New York).

J. Durbin and G. S. Watson. (1950). Testing for Serial Correlation in Least Squares Regression. I, Biometrica 37, pp. 409-428.

J. Durbin and G. S. Watson. (1951). Testing for Serial Correlation in Least Squares Regression. II, Biometrica 38, pp. 159-177.

M. Eaton. (1976). A Maximization Problem and its Application to Canonical Correlation, J. Multivar. Anal. 6, pp. 422-425.

A. Einstein, B. Podolsky and N. Rosen. (1935). Can Quantum Mechanical Description of Reality be Considered Complete?, Phys. Rev. 47, pp. 777-780.

K. Fan. (1966). Some Matrix Inequalities, Abh. Math. Sem. Univ. Hamburg 29, pp. 185-196.

H. Föllmer and A. Schied. (2004). Stochastic Finance, (de Gruyter, Berlin).

G. Golub and C. F. Van Loan. (1987). Matrix Computations, (Johns Hopkins Univ. Press).

T. Goodman, S. Lee and W. Tang. (1993). Wavelets in Wandering Subspaces, Trans. Amer. Math. Soc. 338, pp. 639-654.

G. Gorton. (2008). The Panic of 2007, report prepared for the Federal Reserve Bank of Kansas City, Jackson Hole Conference, August 2008. Available online (90 pp.).

W. Greub and W. Rheinboldt. (1959). On a Generalization of an Inequality of L. V. Kantorovich, Proc. Amer. Math. Soc. 10, pp. 407-415.

K. Gustafson. (1973a). Some Essentially Selfadjoint Dirac Operators with Spherically Symmetric Potentials, Israel J. Math. 14, pp. 63-75 (with P. Rejto).

K. Gustafson. (1981a). Partial Inner Product Spaces and Semi-Inner Product Spaces, Adv. Math. 41, pp. 281-300 (with J. P. Antoine).

K. Gustafson. (1981b). Timestep Control for the Numerical Solutions of InitialBoundary Value Problems, Quantum Mechanics in Mathematics, Chemistry, and 
Physics, Gustafson K. and Reinhardt W. (eds.), (Plenum Press, NY), pp. 407-414 (with J. Gary and H. Tadjeran).

K. Gustafson. (1982a). Exact Solutions and Ignition Parameters in the Arrhenius Conduction Theory of Gaseous Thermal Explosion, ZAMP 33, pp. 391-405 (with B. Eaton).

K. Gustafson. (1983b). Divergence-Free Bases for Finite Element Schemes in Hydrodynamics, SIAM J. Numer. Anal. 20, pp. 697-721. (with R. Hartman).

K. Gustafson. (1985a). A New Method for Computing Solenoidal Vector Fields on Arbitrary Regions, Int. J. Numer. Meth. Fluid 5, pp. 763-783 (with K. Halasi, D. P. Young).

K. Gustafson. (1987a). Kolmogorov Systems and Haar Systems, Colloq. Math. Soc. Janos Bolyai 49, pp. 401-416 (with R. K. Goodrich).

K. Gustafson. (1988a). Multigrid Localization and Multigrid Grid Generation for the Computation of Vortex Structures and Dynamics of Flows in Cavities and About Airfoils, in Multigrid Methods, McCormickS., (ed.),(Dekker), pp. 229-250 (with R. Leben).

K. Gustafson. (1990a). Vortex Methods and Vortex Motion, (SIAM Publications, Philadelphia) (with J. Sethian).

K. Gustafson. (1990b). Counting the Number of Solutions in Combustion and Reactive Flow Problems, J. Appl. Math. Phys. 41, pp. 558-578 (with E. Ash, B. Eaton).

K. Gustafson. (1991b). Computational Physics, (North Holland, Amsterdam) (with W. Wyss).

K. Gustafson. (1991c). Preconditioned Conjugate Gradient and Finite Element Methods for Massively Data-Parallel Architectures, Comput. Phys. Commun. 65, pp. 253-267 (with N. Sobh).

K. Gustafson. (1992a). Lift and Thrust Generation by an Airfoil in Hover Modes, Comput. Fluid Dynam. J. 1, pp. 47-57 (with R. Leben, J. McArthur).

K. Gustafson. (1994d). Risk in Portfolio Management, Asea Brown Boveri (ABB) Internal Report, p. 18.

K. Gustafson. (1998h). Haar Wavelets and Differential Equations, Differential Equations 34, pp. 829-832 (with I. Antoniou).

K. Gustafson. (1999f). Introduction to Partial Differential Equations and Hilbert Space Methods, 3rd edn. (Revised), (Dover Publications, Mineola, NY).

K. Gustafson. (1999g). Wavelets and Stochastic Processes, Math. Comput. Simulat. 49, pp. 81-104 (with I. Antoniou).

K. Gustafson. (1999h). Parallel Computing Forty Years Ago, Math. Comput. Simulat. 51, pp. 47-62.

K. Gustafson. (2000e). The Time Operator of Wavelets, Chaos, Solitons, and Fractals 11, pp. 443-452 (with I. Antoniou).

K. Gustafson. (2000f). A 9th Derivation of the Black-Scholes Equation, preprint.

K. Gustafson. (2002c). Distinguishing Discretization and Discrete Dynamics, with Application to Ecology, Machine Learning, and Atomic Physics, in Structure 
and Dynamics of Nonlinear Wave Phenomena, Tanaka M. (ed.), RIMS Kokyuroku, Vol. 1271, Kyoto, Japan.

K. Gustafson. (2002d). Time-Space Dilations and Stochastic-Deterministic Dynamics, in Between Chance and Choice, Atmanspacher H. and Bishop R. (eds.), (Imprint Academic, UK), pp. 115-148.

K. Gustafson. (2007c). Wavelets and Expectations: A Different Path to Wavelets, in Harmonic, Wavelet and p-adic Analysis, Chuong N., Egorov Y., Khrennikov A., Meyer Y. and Mumford D., (eds.), (World Scientific), pp. 5-22.

K. Gustafson. (2010f). Experiences and Insights in Mathematical Finance, J. Shanghai Finance Univ. No. 4, pp. 35-41 (In Chinese).

K. Gustafson. (2011d). Normal Degree, Revisited. In preparation.

K. Gustafson. (2011e). The Crossing of Heaven: Memoirs of a Mathematician, (SpringerHeidelberg), publication date 28 Dec. 2011.

P. Halmos. (1967). A Hilbert Space Problem Book, (D. Van Nostrand, Princeton, NJ).

E. Hille and R. S. Phillips. (1957). Functional Analysis and Semigroups, Colloq. Publ. Amer. Math. Soc., (AMS Providence, RI).

R. Horn and C. Johnson. (1985). Matrix Analysis, (Cambridge).

G. Jaeger and K. Ann. (2008). Entanglement Sudden Dealth in a Qubit-Qutrit System. Phys. Lett. A 372, pp. 579-583.

N. Jewell, P. Bloomfield and F. Bartmann. (1983). Canonical Correlations of Past and Future for Time Series: Bounds and Computation, Ann. Statist. 11, pp. 848-855.

S. Johnson. (2009). The Quiet Coup, The Atlantic Magazine, May issue.

I. Kaporin. (1990). New Convergence Results and Preconditioning Strategies for the Conjugate Gradient Method, Numer Lin. Alg. with Applic. 1, 179-210.

T. Kato. (1976). Perturbation Theory for Linear Operators, (Springer, Berlin), 13 pp.

M. Knill and R. Laflamme. (1997). Theory of Quantum Error-Correcting Codes. Phys. Rev. A 55, pp. 900-911.

M. Kus and K. Zyczkowski. (2001). Geometry of Entangled States, Phys. Rev. A 63, 032307, 13 pp.

N. Levan and C. Kubrusly. (2003). A Wavelet "Time-Shift-Detail" Decomposition, Math. Comput. Simulat. 63, pp. 73-78.

N. Levan and C. Kubrusly. (2004). Time-Shifts Generalized Multiresolution Analysis over Dyadic-Scaling Reducing Subspaces, Int. J. Wavelets Multiresolut. Inf. Process. 2, pp. 1-12.

M. Lewis. (2010). The Big Short, (Norton).

J. Liesen and P. Saylor. (2005). Orthonormal Hessenberg Reduction and Orthogonal Krylov Subspace Bases, SIAM J. Numer Anal. 42, pp. 2148-2158.

D. Luenberger. (1973). Introduction to Linear and Nonlinear Programming, (AddisonWesley Publ. Co.).

G. Lumer and R. S. Phillips. (1961). Dissipative Operators in a Banach Space, Pacific J. Math. 11, pp. 679-698. 
D. MacKenzie and Y. Millo. (2003). Constructing a Market, Performing Theory: The Historical Sociology of a Financial Derivatives Exchange, Amer. J. Sociol. 109, pp. 107-145.

W. Magnus. (1974). Noneuclidean Tesselations and their Groups, (Academic Press, New York).

A. Marshall and I. Olkin. (1990). Matrix Versions of the Cauchy and Kantorovich Inequalities, Aequationes Math. 40, pp. 89-93.

C. McCarthy. (1967). C $\mathrm{p}$, Israel J. Math 5, pp. 249-271.

C. Mendl and M. Wolf. (2009). United Quantum Channels - Convex Structure and Revivals of Birkhoff's Theorem. Commun. Math. Phys. 289, pp. 1057-1086.

N. D. Mermin. (2007). Quantum Computer Science: An Introduction, (Cambridge Univ. Press, Cambridge).

R. C. Merton. (1990). Continuous Time Finance. (Basil Blackwell, Oxford, UK).

C. Meyer. (2000). Matrix Analysis and Applied Linear Algebra, (SIAM Publications, Philadelphia).

B.S. Nagy and C. Foias. (1970). Harmonic Analysis of Operators in Hilbert Space, (North Holland).

E. Nelson. (1964). Feynman Integrals and the Schrodinger Equation, J. Math. Phys. 5, pp. 332-343.

M. Nielsen and I. Chuang. (2000). Quantum Computation and Quantum Information, (Cambridge Press, Cambridge).

I. Olkin. (1981). Range Restrictions for Product-Moment Correlation Matrices, Psychometrika 46, pp. 469-472.

R. Ortega and M. Santander. (2002). Trigonometry of "complex Hermitian"-Type Homogeneous Symmetric Spaces, J. Phys. A: Math. Gen. 35, pp. 7877-7917.

S. Patterson. (2010). The Quants. (Random House).

R. Penrose. (2005). The Road to Reality, (Alfred Knopf, New York).

M. Pourahmadi. (2001). Foundations of Time Series Analysis and Prediction Theory, (Wiley, New York).

C. R. Rao and C. V. Rao. (1987). Stationary Values of the Product of Two Rayleigh Quotients: Homologous Canonical Correlations, Sankhya 49B, pp. 113-125.

B. Schwarzschild. (1999). At Last We have an Undisputed Observation of "Direct" CP Violation in Kaon Decay. Physics Today, May, 1999, pp. 17-19.

B. Schwarzschild. (2003). Antineutrinos from Distant Reactors Simulate the Disappearance of Solar Neutrinos, Physics Today, March, pp. 14-16.

J. Stampfli. (1970). The Norm of a Derivation, Pacific J. Math. 33, pp. 737-747.

G. Strang. (1962). Eigenvalues of Jordan Products, Amer. Math. Monthly 69, pp. 37-40.

N. Touzi. (1999). American Option Exercise Boundary when the Volatility Changes Randomly, Appl. Math. Optimiz. 39, pp. 411-422.

G. 't Hooft. (2002). Determinism Beneath Quantum Mechanics, ArXiv: quant-ph/ $0212095 \mathrm{v} 1$. 
R. Varga. (1962). Matrix Iterative Analysis, (Prentice Hall).

J. Von Neumann. (1941). Distribution of the Ratio of Mean Square Successive Difference to the Variance, Annals of Math. Stat. 12, pp. 367-395.

J. von Neumann and O. Morgenstern. (1944). Theory of Games and Economic Behavior, (Princeton Univ. Press).

J. Von Neumann, R. Kent, H. Bellinson and B. Hart. (1941). The Mean Square Successive Difference, Annals of Math. Stat. 12, pp. 153-162.

S. G. Wang and S. C. Chow. (1994). Advanced Linear Models, (Marcel Dekker, New York).

S. Wang and W. Ip. (2000). A Matrix Version of the Wielandt Inequality and its Applications to Statistics, Lin. Alg. and Applic. 296, pp. 171-181.

E. Wigner. (1970). On Hidden Variables and Quantum Mechanical Probabilities, Amer. J. Phys. 38, pp. 1005-1009.

P. Wilmott. (1998). Derivatives: The Theory and Practice of Financial Engineering, ( John Wiley \& Sons, Chichester).

H. Yanai, S. Putanen, K. Ito and H. Ishii. (2008). Some Extensions on the Ranges of a Correlation Matrix and its Inverse Matrix, preprint, $14 \mathrm{pp}$.

K. Yosida. (1968). Functional Analysis, (Springer, Berlin).

D. M. Young. (1971). Iterative Solutions of Large Linear Systems, (Academic Press).

R. Zvan, P. Forsyth and K. Vetzal. (1998). Penalty Methods for American Options with Stochastic Volatility. J. Comput. Appl. Math. 91, pp. 199-218. 


\section{Index}

$\phi(A), 2$

absolute condition number, 64

Accardi, L., 123

accretive operator, 5

additive Schwarz, 62

ADI, 60

Alfred Haar Memorial Conference, 69

Alfred Haar's 1910 dissertation, 72 angles, 1, 2, 6, 7, 58, 186

Antieigenmatrices, 115

antieigenmatrix, 65

antieigenvalue, 1, 2, 6, 33, 34

antieigenvector, 1, 6, 7, 30, 31, 38, 40

Antieigenvector Pair Angle, 44, 85

Antieigenvector-Breaking, 143

antieigenvectors, 7, 30, 31, 33, 39, 84

Antoine, J.P., 25

Ash, E., 54

Asplund, E., 49

BA, $4,14,63$

Basis Trigonometry, 78

Bell's inequalities, 123

Bell's inequality, 153

Berlin, 164

bid-ask gap, 159

Black-Scholes, 23

Black-Scholes PDE, 157

Bloch sphere, 154

Breckenridge, 66

Brussels, 69

Budapest, 69
Calvert, B., 19

Canonical correlations, 105, 114

Chaitin-Chatelin, F., 64

coarse-graining, 71

combinatorial viewpoint, 39

computational experience, 53

Computational Fluid Dynamics, 157

condition number, 43, 63, 188

conjugate gradient, 67

contraction semigroup, 2, 3, 5, 16, 17,

18,21

completely nonunitary, 88

Convexity, 26

$\cos \phi(A), 4$

Davenport, J., 159

Davis, C., 13, 50, 184

De Moivre's theorem, 83

Decoherence, 146

Dirichlet boundary conditions, 82

dispersiveness, 22

dissipativeness, 22

Domain Decomposition, 61

Dorroh, J., 18

Dubrovnik, ix, 55

Dubrovnik Workshop, 53

Durbin-Bloomfield-Watson-Knott lower bound, 92

Eaton, B., 54

eigenvalue, 1, 2

eigenvectors, 1

Elementary Particles, 142

Entanglement, 144 
Entropy, 145

EPR, 125

Euler Equation, 33, 36, 41, 101

Extended Operator Trigonometry, 47

financial crashes, 163

Financial Instruments, 155

Fréchet derivative, 64

frame, 74,89

frame operator, 74

maximal turning angle, 75

Frobenius norm, 64

Fundamental Theorem of Linear

Algebra, 203

general spinor, 141

golden mean, 202

Gradient Descent, 54

Gram matrix, 46

Gram matrix determinant, 47, 198

graph norm, 23

Green's function, 16

Greub-Rheinboldt bounds, 21

Haar Basis, 73

Haar wavelet, 73, 89

Halasi, K., 54

Hartman, R., 54

heat equation, 16

Hess, P., 189

Higher Antieigenvalues, 39

Hille-Yosida, 16

Hotelling correlation, 108

Hydrogen, 18

imaginary operator trigonometry, 186 Inefficiency Equation, 101

inequality-equalities, 129, 153, 191

Infinitesimal Generators, 17

Initial Value Problems, 2, 16

interaction-antieigenvalues, 187

intra-angle, 84

Irreversibility, 86

Ito, K., 101, 118

IWMS 2005, 91
Jackson Hole, Wyoming, 70

Jordan canonical form, 213

Kantorovich, L., 50, 53, 65

Kantorovich-Wielandt inequality, 55

Kaporin, I., 188

Kato, T., 17

Khatree, R., 13

Kolmogorov systems, 70, 88

Kolmogorovian probability, 124

Krein, M.G., 13, 46, 47, 49

Lagrange multiplier methods, 42

Lagrangian variational methods, 101

Laplacian, 23, 58, 82

Laplacian operator, 16

Leben, R., 54

Les Treilles in Provence, 123

light cone, 135

Littlewood-Paley basis, 79

Los Angeles, ix, 27, 49

Lyapunov Stability, 85

Markov process, 22

matrix products, 4

matrix statistics, 34

Maybee, J., 27

McArthur, J., 54

McCarthy, C., 20

Min-Max, 27

Min-Max Theorem, 5, 27, 31, 104, 137

Minimum Residual, 56

Mirman, B., 13, 50

Model Problem, 58

Morse theory, 45

most-turned vectors, 6

Multiplicative Perturbation, 18, 86

multiplicatively perturb, 3

multiresolution analyses, 71

NAPA Preconditioning Conference, 64

Navier elasticity, 55

Nelson, E., 17

Netherlands, 66

New Zealand, 91

noncommuting, 4 
normal operators, 184

numerical range, 4, 50

oblique projections, 14

one-parameter unitary group, 86

operator angle, 58

operator trigonometry, 2

operator turning, 1

optimal, 57,58

Optimum, 31, 32

ordinary differential equations, 23

orthonormal basis, 75, 79

Pascal's triangle, 83

Penrose, R., 124

Poisson-Neumann boundary value problem, 101

polar form, 48

Portfolio, 175

positivity of matrix products, 20

Preconditioning, 63

Prediction Theory, 112

Ptak, V., 49

Puntanen, S., 117

quadratic equation, 43

Quantos, 167

Quantum Computing, 132

Quantum Fidelity, 149

Quantum Spin Identities, 129

Quantum States, 144

quark mixing, 143

Rao, C.R., 13, 91, 105, 117

Rao, D., 13, 45, 46, 49

Rayleigh eigenvalue, 90

Rayleigh quotient, 14, 105

Rayleigh variational quotients, 33

Rayleigh-Ritz theory, 26

re-inner-product, 184

reducing subspace, 41

Rejto, P., 20

Rellich, F., 17

Richardson iteration, 74, 77

Richardson Relaxation, 57

Risk axioms, 160
Sato, K., 19, 21

Schneider, H., 49, 55

Schrödinger equation, 86

Schrodinger, E., 17, 18

Schwarz's inequality, 14, 32

second antieigenvector, 7

Seddighin, M., 13, 45, 184

Seguin, T., 159

semigroup, 2, 23, 86

Seneta, E., 91

Shanghai Finance University, 160

Shisha-Mond inequality, 190, 201

$\sin \phi(A), 5,8,9,194$

singular value, 48,64

skew antieigenvalues, 44

slant antieigenvalues, 186

Sobh, N., 53

Solvay Institute, 69, 123

Solvers, 56

SOR method, 58

spacetime angle, 135

spectral radius, 45,59

spectral representation, 14

spectral theory, 1

spin model, 124

Spread, 172

Stampfli, J., 13, 21

Statistical Efficiency, 93

Statistics, 91

Statistics Inequalities, 107

Stimmel, E., 14

Stone-Von Neumann Theorem, 89

Strang, G., 13, 21

Sturm-Liouville boundary value problem, 81

Styan, G., 117

suddenly incomplete market, 164

symmetric antieigenvalue, 186

"symplectic" representation, 197

Tadjeran, H., 54

't Hooft, G., 200

Thorp, E., 182

Time operator, 69,72

Toeplitz-Hausdorff Theorem, 50

Toronto, 50 


\section{Antieigenvalue Analysis}

total operator trigonometry, 186

"total" turning angle, 132

Triangle Inequality, 46

Trigonometry of efficiency, 93

Triple Commute, 63

twisting vector, 80

Twistors, 135, 138

twistvectors, 49

University of Minnesota, 20, 21

Volatility, 177

Von Neumann, J., 98, 118 wandering subspace, 69,88

wavelet, 70, 88

Wavelet Reconstruction, 76

Wielandt, H., 13, 49

Wigner, 125

Wigner inequality-equality, 153

Wisconsin, 49, 55

Young, D.M., 58, 60

Young, D.P., 54

Zwahlen, B., 19 